\title{
Spatially resolved carbon and oxygen isotopic ratios in NGC 253 using optically thin tracers
}

\author{
S. Martín ${ }^{1,2}$, S. Muller ${ }^{3}$, C. Henkel ${ }^{4,5}$, D. S. Meier ${ }^{6,7}$, R. Aladro ${ }^{4}$, \\ K. Sakamoto ${ }^{8}$, and P. P. van der Werf ${ }^{9}$ \\ ${ }^{1}$ European Southern Observatory, Alonso de Córdova, 3107, Vitacura, Santiago 763-0355, Chile \\ e-mail: smartin@eso.org \\ 2 Joint ALMA Observatory, Alonso de Córdova, 3107, Vitacura, Santiago 763-0355, Chile \\ 3 Department of Space, Earth and Environment, Chalmers University of Technology, Onsala Space Observatory, \\ 43992 Onsala, Sweden \\ ${ }^{4}$ Max-Planck-Institut für Radioastronomie, Auf dem Hugel 69, 53121 Bonn, Germany \\ 5 Astron. Dept., King Abdulaziz University, PO Box 80203, Jeddah 21589, Saudi Arabia \\ 6 New Mexico Institute of Mining and Technology, 801 Leroy Place, Socorro, NM 87801, USA \\ 7 National Radio Astronomy Observatory, PO Box O, 1003, Lopezville Road, Socorro, NM 87801, USA \\ 8 Institute of Astronomy and Astrophysics, Academia Sinica, PO Box 23-141, 10617 Taipei, Taiwan \\ ${ }^{9}$ Leiden Observatory, Leiden University, PO Box 9513, 2300 RA Leiden, The Netherlands
}

Received 22 January 2019 / Accepted 6 March 2019

\begin{abstract}
Context. One of the most important aspects of modern astrophysics is related to our understanding of the origin of elements and chemical evolution in the large variety of astronomical sources. Nuclear regions of galaxies undergo heavy processing of matter, and are therefore ideal targets to investigate matter cycles via determination of elemental and isotopic abundances.

Aims. To trace chemical evolution in a prototypical starburst environment, we spatially resolve carbon and oxygen isotope ratios across the central molecular zone (CMZ; full size $\sim 600 \mathrm{pc})$ in the nearby starburst galaxy NGC 253.

Methods. We imaged the emission of the optically thin isotopologues ${ }^{13} \mathrm{CO}, \mathrm{C}^{18} \mathrm{O}, \mathrm{C}^{17} \mathrm{O},{ }^{13} \mathrm{C}^{18} \mathrm{O}$ at a spatial resolution $\sim 50 \mathrm{pc}$, comparable to the typical size of giant molecular associations. Optical depth effects and contamination of ${ }^{13} \mathrm{C}^{18} \mathrm{O}$ by $\mathrm{C}_{4} \mathrm{H}$ are discussed and accounted for to derive column densities.

Results. This is the first extragalactic detection of the double isotopologue ${ }^{13} \mathrm{C}^{18} \mathrm{O}$. Derived isotopic ratios ${ }^{12} \mathrm{C} /{ }^{13} \mathrm{C} \sim 21 \pm 6,{ }^{16} \mathrm{O} /{ }^{18} \mathrm{O} \sim$ $130 \pm 40$, and ${ }^{18} \mathrm{O} /{ }^{17} \mathrm{O} \sim 4.5 \pm 0.8$ differ from the generally adopted values in the nuclei of galaxies.

Conclusions. The molecular clouds in the central region of NGC 253 show similar rare isotope enrichment to those within the CMZ of the Milky way. This enrichment is attributed to stellar nucleosynthesis. Measured isotopic ratios suggest an enhancement of ${ }^{18} \mathrm{O}$ as compared to our Galactic centre, which we attribute to an extra ${ }^{18} \mathrm{O}$ injection from massive stars. Our observations show evidence for mixing of distinct gas components with different degrees of processing. We observe an extra molecular component of highly processed gas on top of the already proposed less processed gas being transported to the central region of NGC 253. Such a multicomponent nature and optical depth effects may hinder the use of isotopic ratios based on a spatially unresolved line to infer the star formation history and/or initial stellar mass function properties galaxy nuclei.
\end{abstract}

Key words. line: identification - ISM: molecules - ISM: abundances - Galaxy: abundances - galaxies: individual: NGC 253 galaxies: starburst

\section{Introduction}

Interstellar isotope ratios carry essential information on the processes of nucleosynthesis in the hot and dense interior of stars. Measuring isotopic abundances such as ${ }^{12} \mathrm{C} /{ }^{13} \mathrm{C}$ and ${ }^{18} \mathrm{O} /{ }^{17} \mathrm{O}$ in the nuclear regions of starburst galaxies can therefore reveal the fingerprint of their star formation history. Isotopic ratios can be also be related to the relative contribution of high-mass to intermediate-mass stellar processing (Zhang et al. 2015). Thus these ratios can serve as tracers of the chemical evolution of the interstellar medium (ISM) due to stellar processing. The recent work by Romano et al. (2017) has attempted to model the available isotopic ratios observed in galaxies as a function of the star forming history and the stellar initial mass function (IMF). Based on their comparison of observations and model results they find evidence of a top-heavy IMF responsible for the observed ratios in starburst galaxies.
However, measuring isotopic ratios in astronomical sources is not always straightforward. At optical wavelengths, such measurements are difficult or impossible owing to the blending of atomic isotope lines and isotope specific obscuration from dust grains. Isotopic measurements of atomic carbon in the farinfrared and carbon monoxide in the near-infrared have been obtained (i.e. Goto et al. 2003, and references therein). On the other hand, observations of molecular isotopologues at radio to submillimeter wavelengths have been proven most successful within the Galaxy, towards the local extragalactic ISM (see Martín et al. 2010; Henkel et al. 2014; Romano et al. 2017, and references therein), and all the way to high redshift molecular absorbers (i.e., Muller et al. 2011; Wallström et al. 2016).

In this paper we focus on the isotopic ratio of ${ }^{12} \mathrm{C}$ to ${ }^{16} \mathrm{O}$, as primary products of stellar nucleosynthesis, and the rarer ${ }^{13} \mathrm{C}$, ${ }^{18} \mathrm{O}$, and ${ }^{17} \mathrm{O}$, as secondary nuclear products from primary seeds (Meyer 1994; Wilson \& Matteucci 1992; Wilson \& Rood 1994). 
We note that, primary production of ${ }^{13} \mathrm{C}$ and ${ }^{18} \mathrm{O}$ is predicted for fast-rotating low metallicity massive stars and intermediatemass stars climbing the asymptotic giant branch (Chiappini et al. 2008; Karakas \& Lattanzio 2014; Limongi \& Chieffi 2018), but the former results in a relatively small enrichment while the latter will not be relevant for the overall galactic scales involved in this work. In this context, when referring to enrichment we mean rare isotopologues enrichment, and therefore lower ${ }^{12} \mathrm{C} /{ }^{13} \mathrm{C},{ }^{16} \mathrm{O} /{ }^{18} \mathrm{O}$, and ${ }^{18} \mathrm{O} /{ }^{17} \mathrm{O}$ isotopic ratios. As compiled in Wilson \& Rood (1994) and Henkel et al. (1994), the generally adopted values in the nuclei of low luminosity local starbursts are ${ }^{12} \mathrm{C} /{ }^{13} \mathrm{C} \sim 40,{ }^{16} \mathrm{O} /{ }^{18} \mathrm{O} \sim 200$, and ${ }^{18} \mathrm{O} /{ }^{17} \mathrm{O} \sim 8$ (Sage et al. 1991; Henkel et al. 1993; Henkel \& Mauersberger 1993). These ratios are derived from C- and O-bearing molecular species commonly observed in the ISM, such as CO, CN, CS, $\mathrm{HCN}, \mathrm{HNC}$, and $\mathrm{HCO}^{+}$, which, despite not suffering from line blending between the main and rare isotopologues, may actually be biased by optical depth effects. These limitations result in most of the cited ratios to be lower limits. To circumvent this problem, it is sometimes possible to turn to rare isotopologues involving another element, for example studying the double ratio ${ }^{12} \mathrm{C}^{34} \mathrm{~S} /{ }^{13} \mathrm{C}^{32} \mathrm{~S}$, but this adds potential problems due to poor understanding of the extra isotopic ratio (in this example, ${ }^{32} \mathrm{~S} /{ }^{34} \mathrm{~S}$, Henkel et al. 2014; Meier et al. 2015).

Recently, Henkel et al. (2014) used high quality spectra of $\mathrm{CN}$ towards the starburst galaxy NGC 253 to revisit its ${ }^{12} \mathrm{C} /{ }^{13} \mathrm{C}$ ratio. Thanks to the hyperfine splitting of $\mathrm{CN}$, it is possible to estimate the optical depth of the main isotopologue. The resulting ${ }^{12} \mathrm{C} /{ }^{13} \mathrm{C} \sim 40 \pm 10$ confirmed some of the previous estimates. An accurate measurement of the ${ }^{12} \mathrm{C} /{ }^{13} \mathrm{C}$ ratio also becomes important because this ratio is often used to derive other atomic isotopic ratios based on observations of the optically thinner ${ }^{13} \mathrm{C}$ bearing isotopologues (i.e., Martín et al. 2005).

However, there are several non-local thermodynamic equilibrium (non-LTE) effects that can affect the observed molecular line ratios based on optically thick species (Meier \& Turner 2001; Meier et al. 2015). As pointed out by Wilson \& Rood (1994), in order to minimize effects such as optical thickness of the emission, excitation differences due to resonant photon trapping and/or selective photodissociation, we would rather choose the observation of optically thin isotopologues such as $\mathrm{C}^{18} \mathrm{O}$ and ${ }^{13} \mathrm{C}^{18} \mathrm{O}$. Despite the weakness of the emission of these isotopologues, observations have been successfully performed across the Galaxy (Langer \& Penzias 1990; Ikeda et al. 2002), but are very challenging in extragalactic sources. High sensitivity observations with the IRAM 30 m telescope were carried out by Martín et al. (2010) in an attempt to detect ${ }^{13} \mathrm{C}^{18} \mathrm{O}$ towards the nearby starburst galaxies NGC 253 and M 82. The resulting tentative detection towards NGC 253 yielded a lower limit of ${ }^{12} \mathrm{C} /{ }^{13} \mathrm{C} \gtrsim 60$, which is comparatively higher than previous estimates.

Another important effect to take into account is that of isotopic fractionation (Watson 1977; Langer et al. 1984; Wilson \& Rood 1994; Röllig \& Ossenkopf 2013; Szúcs et al. 2014; Roueff et al. 2015), in which the unbalance of exothermic isotope exchange chemical reactions may favour the enhancement of the rarer isotopologues. The most relevant isotope barrierless fractionation reactions for the carbon monoxide species studied in this paper are written as

$$
\begin{aligned}
& { }^{13} \mathrm{C}^{+}+\mathrm{CO} \rightarrow \mathrm{C}^{+}+{ }^{13} \mathrm{CO}+\Delta E_{1} \\
& { }^{13} \mathrm{C}^{+}+\mathrm{C}^{18} \mathrm{O} \rightarrow \mathrm{C}^{+}+{ }^{13} \mathrm{C}^{18} \mathrm{O}+\Delta E_{2} \\
& { }^{18} \mathrm{O}^{+}+\mathrm{CO} \rightarrow \mathrm{O}^{+}+\mathrm{C}^{18} \mathrm{O}+\Delta \mathrm{E}_{3}
\end{aligned}
$$

where the heats of the reaction are $\Delta E_{1}=35 \mathrm{~K}, \Delta E_{2}=36 \mathrm{~K}$, and $\Delta E_{3}=38 \mathrm{~K}$, respectively (Langer et al. 1984; Loison et al. 2019). The equilibrium constant, and therefore the relation between the measured and observed isotopologue ratios is proportional to $\exp \left(\Delta E / T_{\text {kin }}\right)$, where $T_{\text {kin }}$ is the kinetic temperature of the gas (Wilson \& Rood 1994). Therefore, these reactions are relevant in the cold ISM.

Based on the observed gradients in the Galaxy by Milam et al. (2005), and Romano et al. (2017) concluded that carbon fractionation effects are negligible for the bulk of the gas in galaxies. However, Jiménez-Donaire et al. (2017) invoked fractionation as a contributor to the ${ }^{13} \mathrm{CO} / \mathrm{C}^{18} \mathrm{O}$ gradient with galactocentric radius in a sample of nearby galaxies.

Given that the central molecular zone (CMZ) of NGC 253 has a relatively high kinetic temperature (Ott et al. 2005; Mangum et al. 2013, 2019; Krips et al. 2016), fractionation effects should be negligible in gas phase chemistry. Since we are not able to provide sensible constraints on fractionation issues in the framework of this paper, we therefore simply assume that our measured isotopologue ratios reflect true isotopic ratios.

The bright molecular emitting CMZ of the nearby starburst galaxy NGC 253 (Martín et al. 2006; Aladro et al. 2015) is ideally suited for high resolution studies of the isotopic ratio distribution in an extragalactic environment. At a distance of 3.5 Mpc (Rekola et al. 2005; Mouhcine et al. 2005), it can be easily resolved by interferometric observations at giant molecular cloud scales of a few tens of pc. Within the CMZ of the Milky Way, inhomogeneities in the isotopic ratio distribution have been found and attributed to accretion of material from the outer disc (Riquelme et al. 2010).

In this paper, we aim at shedding light on discrepancies between the ratios derived from low resolution single dish observations, and to study the spatial distribution of the carbon and oxygen isotopic ratios in an extragalactic starburst region. We present high angular resolution and high sensitivity observations of four rare carbon monoxide isotopologues, ${ }^{13} \mathrm{CO}, \mathrm{C}^{18} \mathrm{O}, \mathrm{C}^{17} \mathrm{O}$, ${ }^{13} \mathrm{C}^{18} \mathrm{O}$, reporting the first firm detection of the double isotopologue ${ }^{13} \mathrm{C}^{18} \mathrm{O}$ in an extragalactic environment.

\section{Observations}

The observations were carried out with the Atacama Large Millimeter and Submillimeter Array (ALMA) under the Cycle 4 project 2016.1.00292.S (P.I. S. Martín). The observations consisted of two receiver tunings. The first tuning set-up had spectral windows centred at $91.0,92.8,102.9$, and $104.7 \mathrm{GHz}$ and aimed at covering the $J=1-0$ transition of ${ }^{13} \mathrm{C}^{18} \mathrm{O}$ at $104.711 \mathrm{GHz}$. The second tuning set-up had spectral windows centred at 97.9, $99.6,110.0$, and $111.9 \mathrm{GHz}$ and aimed to cover the $J=1-0$ transitions of ${ }^{13} \mathrm{CO}(110.201 \mathrm{GHz}), \mathrm{C}^{18} \mathrm{O}(109.782 \mathrm{GHz})$, and $\mathrm{C}^{17} \mathrm{O}$ $(112.359 \mathrm{GHz})$. In both cases the spectral windows were configured to cover a bandwidth of $1.875 \mathrm{GHz}$ each with a channel spacing of $0.488 \mathrm{MHz}$ (corresponding to $\sim 1.3-1.4 \mathrm{~km} \mathrm{~s}^{-1}$ velocity resolution) after Hanning smoothing.

Observations consisted of a single pointing at the nominal phase centre of $\alpha_{\mathrm{J} 2000}=00^{\mathrm{h}} 47^{\mathrm{m}} 33.182, \delta_{\mathrm{J} 2000}=$ $-25^{\circ} 17^{\prime} 17^{\prime \prime} 148$. The field of view of $\sim 60^{\prime \prime}$ at these frequencies is wide enough to cover the whole CMZ in NGC 253 (see Fig. 1), roughly $\sim 40^{\prime \prime} \times 10^{\prime \prime}(\sim 650 \mathrm{pc} \times 150 \mathrm{pc})$ in size. The observation targeting the faint ${ }^{13} \mathrm{C}^{18} \mathrm{O}$ transition were carried out between January 1 and 3, 2017, with a total on-source time of 170 minutes and unprojected baselines ranging $15-460 \mathrm{~m}$ $(5.2-160 \mathrm{k} \lambda)$ which results in an estimated maximum recoverable scale of $\sim 15^{\prime \prime}$. The precipitable water vapour (PWV) 

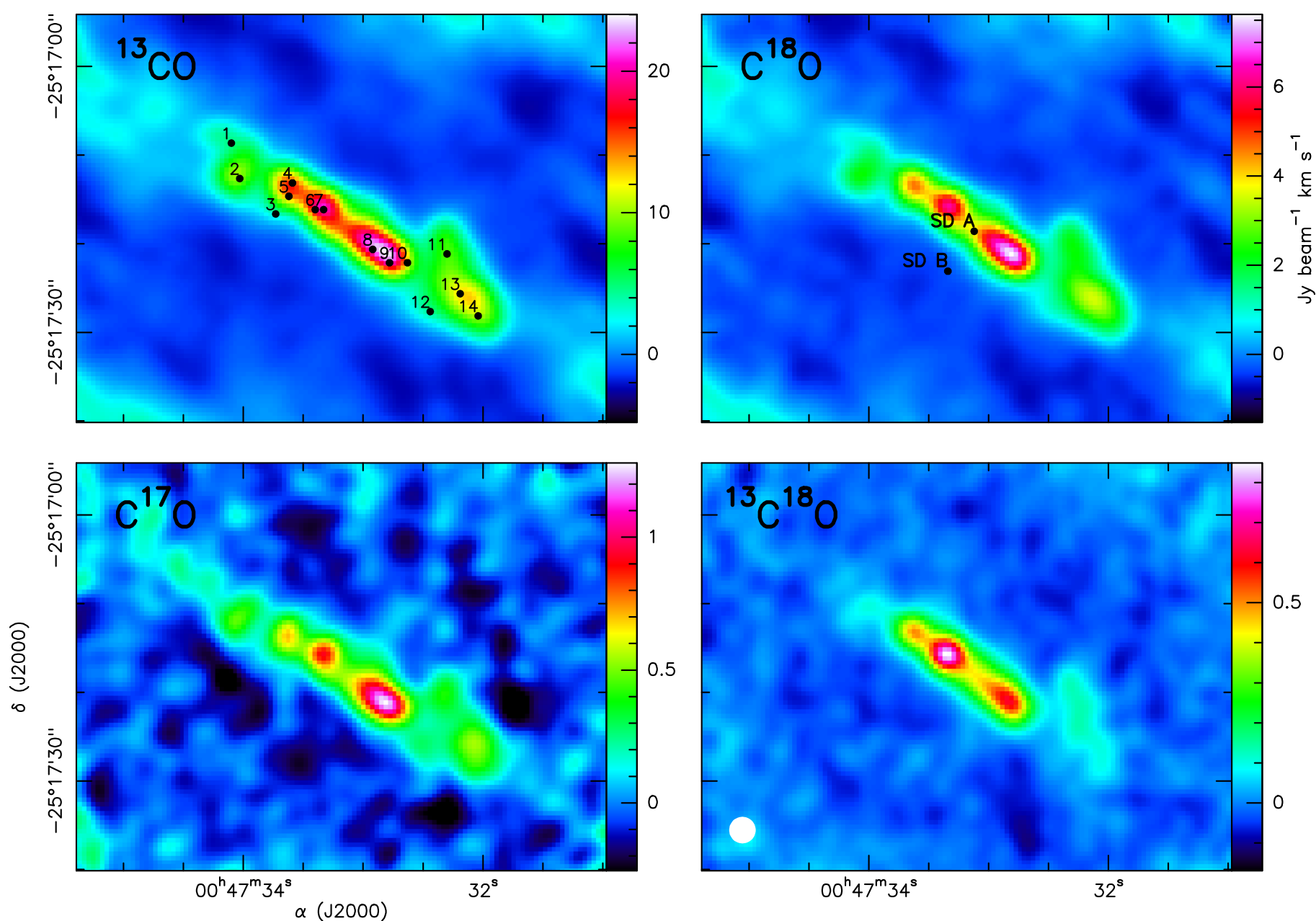

Fig. 1. Integrated intensity images of the $J=1-0$ transitions of all the $\mathrm{CO}$ isotopologues targeted in this work. Line emission is integrated in the velocity range $50-450 \mathrm{~km} \mathrm{~s}^{-1}$. The common beam size of $3^{\prime \prime}(\sim 50 \mathrm{pc})$ is shown as a white circle in the bottom left corner of the ${ }^{13} \mathrm{C}^{18} \mathrm{O}$ map. The emission of ${ }^{13} \mathrm{C}^{18} \mathrm{O}$ is contaminated at the higher velocities in some positions as discussed in Sect. 3.2.1 and shown in Fig. 4. This contamination results in the brighter ${ }^{13} \mathrm{C}^{18} \mathrm{O}$ region at position 7 differing from the brightest spot from the other isotopologues approximately at position 9. Marked positions numbered 1 to 14 indicate those selected for spectra extraction and analysis (Sect. 3.2). Nominal positions of the single dish spectra from the literature used for comparison are labelled SD A and B (Sect. 3.1.1).

conditions were between 4 and $6 \mathrm{~mm}$ during these observations. The second tuning, targeting mainly the brighter $\mathrm{C}^{18} \mathrm{O}$ transition, was observed on December 23, 2016; this tuning had only 12 minutes of on-source integration and unprojected baselines in the range $15-492 \mathrm{~m}(5.5-180 \mathrm{k} \lambda)$ resulting in maximum recoverable scales of $13^{\prime \prime}$. The PWV was $\sim 2.5 \mathrm{~mm}$. In all cases, the number of $12 \mathrm{~m}$ antennas in the array was between 43 and 46.

Observations of the quasars J0006-0623 and J0038-2459 were performed for bandpass and complex gain calibration, respectively. Absolute flux calibration used observations of Neptune and the quasar J0038-2459. Based on the fluxes derived for the bandpass and gain calibrators between the different dates, we conservatively estimate an uncertainty $<10 \%$ between the two frequency set-ups.

Calibration and imaging was performed in CASA version 4.7.2 (McMullin et al. 2007). The continuum emission was subtracted from the visibilities (with the CASA task uvcontsub) using line free channels.

With similar ranges of baseline coverage, the resulting synthesized beams differ only by $\sim 5 \%$ between the two set-ups. Imaging after cleaning was performed with a common circular synthesized beam of $3^{\prime \prime}$ (full width at half maximum; FWHM) for the sake of accurate comparison of flux densities between transitions. This angular resolution is equivalent to $\sim 50 \mathrm{pc}$ at the distance of NGC 253.

Final data cubes of the individual transitions were smoothed to a common $10 \mathrm{~km} \mathrm{~s}^{-1}$ velocity resolution. The achieved rms sensitivities in the velocity smoothed cubes were $\sim 1.5$ and $\sim 5 \mathrm{mJy}^{\text {beam }^{-1}}$, equivalent to $\sim 19$ and $\sim 62 \mathrm{mK}$, for the first and second tuning, respectively.

\section{Results}

\subsection{Integrated flux density maps}

All targeted CO isotopologues were detected at high signal to noise ratio over the whole CMZ in NGC 253. In Fig. 1 we show the integrated flux density maps of the $J=1-0$ transition of all four $\mathrm{CO}$ isotopologues. The map of $\mathrm{C}^{17} \mathrm{O}$ in Fig. 1 at $3^{\prime \prime}$ is similar to that presented by Meier et al. (2015), but the higher spatial resolution allows for resolving the individual GMC complexes in the very central region.

Apart from the difference in line intensities (and therefore also signal to noise ratios), all four isotopologues show a consistent distribution, except for ${ }^{13} \mathrm{C}^{18} \mathrm{O}$ which differs on the 
position of the brighter emission. This is not due to the intrinsic distribution of ${ }^{13} \mathrm{C}^{18} \mathrm{O}$ but to the contamination by other species (Sect. 3.2.1). We tried to generate the ${ }^{13} \mathrm{C}^{18} \mathrm{O}$ integrated map with a mask per channel based on the emission extent observed in ${ }^{13} \mathrm{CO}$. Unfortunately, the closeness of the emission of the molecular contaminant at just $\sim 23 \mathrm{~km} \mathrm{~s}^{-1}$ (Sect. 3.2.1) from the ${ }^{13} \mathrm{C}^{18} \mathrm{O}$ transition made the de-blending impossible in the integrated map. Thus, we preferred to show and use the raw integrated map in the selected velocity range with this limitation in mind when interpreting the map ratios (Sect. 3.1.2).

Carbon and oxygen isotopic ratios can be estimated using the ratio of the carbon monoxide isotopologues as a proxy (Sect. 3.1.2). However, because of the mentioned contamination of the ${ }^{13} \mathrm{C}^{18} \mathrm{O}$ emission, a more accurate measurement can be derived from precise modelling and de-blending of its emission from spectra extracted on selected positions (Sect. 3.2).

\subsubsection{Spatial filtering}

Despite using one of the most compact configurations of the ALMA $12 \mathrm{~m}$ array for the sake of achieving a very low brightness temperature sensitivity (Sect. 2), we still expect that a fraction of the flux is filtered out because of our lack of zero spacing observations.

To evaluate the missing flux due to spatial filtering, we reconstructed our ALMA primary beam corrected cubes with a $23^{\prime \prime}$ beam, equivalent to that of the IRAM $30 \mathrm{~m}$ telescope at the frequency of the $\mathrm{CO}$ isotopologues $J=1-0$ transitions. We extracted the smoothed spectra at the same positions as those from the single dish observations. These positions (indicated in Fig. 1, upper right panel) are SD A, at $\alpha_{\mathrm{J} 2000}=00^{\mathrm{h}} 47^{\mathrm{m}} 33^{\mathrm{s}} .12$, $\delta_{\mathrm{J} 2000}=-25^{\circ} 17^{\prime} 18^{\prime \prime}$, 6 , to match the position of the spectra of the four observed isotopologues by Aladro et al. (2015), and SD B, $\alpha_{\mathrm{J} 2000}=00^{\mathrm{h}} 47^{\mathrm{m}} 33.34, \delta_{\mathrm{J} 2000}=-25^{\circ} 17^{\prime} 23^{\prime \prime}{ }^{\mathrm{s}} 1$, to match the position of the ${ }^{13} \mathrm{C}^{18} \mathrm{O}$ profile reported by Martín et al. (2010). Extracted spectra on the smoothed cubes are shown in Fig. 2 as grey histograms.

Based on our smoothed data cubes we could verify that the published ${ }^{13} \mathrm{C}^{18} \mathrm{O}$ integrated flux by Martín et al. (2010; position SD B) would have been $25 \%$ brighter if measured towards the position from Aladro et al. (2015; position SD A), which is closer to the galaxy centre. This fact may have an impact in the ${ }^{12} \mathrm{C} /{ }^{13} \mathrm{C}$ ratio reported by Martín et al. (2010) as discussed in Sect. 4.2. Moreover, the limited pointing accuracy of the single dish observations may also introduce some uncertainties when comparing different datasets.

The IRAM $30 \mathrm{~m}$ single dish spectra ${ }^{1}$, shown in Fig. 2 in black lines, have been converted to flux density units with a factor of $S / T_{\mathrm{MB}}=5 \mathrm{Jy} \mathrm{K}^{-1}$. Additionally, for ${ }^{13} \mathrm{CO}, \mathrm{C}^{18} \mathrm{O}$, and $\mathrm{C}^{17} \mathrm{O}$, we plot the ALMA spectra (grey histograms) as extracted from the corresponding position in our 23" smoothed cubes, and also multiplied by a factor of 2.5 (shown in red) for the sake of line shape comparison with the single dish data.

We find that, integrated over the velocity range $40-450 \mathrm{~km} \mathrm{~s}^{-1}$, only $28 \%, 26 \%$, and $29 \%$ of the ${ }^{13} \mathrm{CO}$, $\mathrm{C}^{18} \mathrm{O}$, and $\mathrm{C}^{17} \mathrm{O}$ single dish flux is recovered by our ALMA data, respectively. These values are very similar and hint at similar spatial distributions as already pointed out in Sect. 3.1 based on the maps in Fig. 1. However, this is around a factor of 2 lower than the flux recovery of $60 \%$ reported for $\mathrm{C}^{17} \mathrm{O}$ by Meier et al. (2015). Such a difference is due to the different

\footnotetext{
1 http://wwW.iram.es/IRAMES/mainWiki/ Iram30mEfficiencies
}

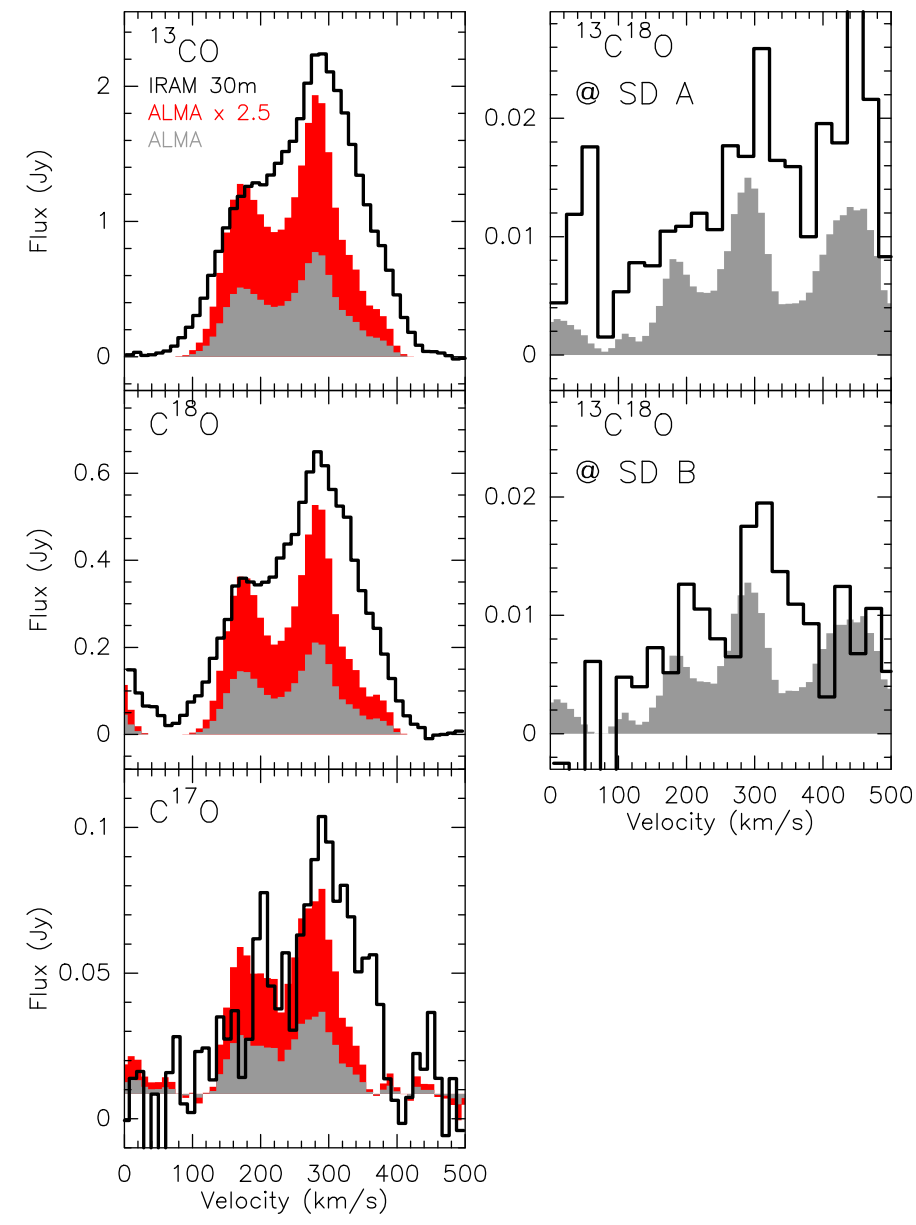

Fig. 2. Spectra measured with the IRAM $30 \mathrm{~m}$ telescope (Aladro et al. 2015) and the ALMA observations presented in this work. ALMA spectra were extracted at the same positions as the single-dish observations from the data cubes smoothed to $23^{\prime \prime}$ resolution. For ${ }^{13} \mathrm{CO}, \mathrm{C}^{18} \mathrm{O}$, and $\mathrm{C}^{17} \mathrm{O}$, the ALMA data are shown both with their actual flux (grey histogram) and multiplied by 2.5 (red histogram) for comparison with the single dish line profiles (black line). For ${ }^{13} \mathrm{C}^{18} \mathrm{O}$, we show the profiles in both SD A and SD B positions in Fig. 1, for comparison with the single dish data from Aladro et al. (2015) and Martín et al. (2010), respectively. See text in Sect. 3.1.1 for details.

single dish integrated flux reported by Henkel et al. (2014; $1.68 \pm 0.15 \mathrm{~K} \mathrm{~km} \mathrm{~s}^{-1}$ ) that Meier et al. (2015) used as a reference and the factor of 2 higher value from Aladro et al. (2015; $3.15 \pm 0.14 \mathrm{~K} \mathrm{~km} \mathrm{~s}^{-1}$ ) we use in this paper. The different single dish fluxes are due to different observed pointing positions. This is reflected by the narrower line width derived by Henkel et al. (2014), mainly targeting the southwestern part of the central ridge, as compared to that by Aladro et al. (2015). When using the same single dish reference, both Meier et al. (2015) and this work recover the same fluxes. We consider our missing flux estimates more robust since they are derived from smoothed data at the same resolution and position as the single dish data. Meier et al. (2015) reported scales sampled up to $18^{\prime \prime}$ (90th percentile), similar to our larger recoverable scales (Sect. 2), which points to significant amounts of gas at larger scales.

For ${ }^{13} \mathrm{C}^{18} \mathrm{O}$, the recovered flux measured is $\sim 60 \%$ and $\sim 45 \%$ for the Martín et al. (2010) and Aladro et al. (2015) positions, respectively. If we consider velocities only up to $350 \mathrm{~km} \mathrm{~s}^{-1}$ to reduce the effect of line contamination at the higher velocities 
(Sect. 3.2.1), the recovered flux yields similar values of $\sim 55 \%$ and $\sim 45 \%$, respectively. Although, the longer integration at this frequency set-up might result in a better inner-UV coverage, the difference in the recovered flux compared to the other isotopologues can be attributed to: $i$ ) significantly varying spatial distribution of ${ }^{13} \mathrm{C}^{18} \mathrm{O}$, which, in principle, we would not expect to be so different from the other rare isotopologues; ii) a higher degree of compactness of the emission of the molecular contaminant (Sect. 3.2.1), which contributes to the measured integrated emission; and more importantly, iii) to the lower signal to noise of this spectral profile affected by both a larger absolute flux uncertainty due to the noise and the extra uncertainty due to the baseline subtraction performed on the single dish spectra (see Fig. 2).

These results point to the uncertainties inherent to single dish observations. In that sense, as pointed out by Meier et al. (2015), the interferometric observations may provide us with a more homogeneous look at the isotopic ratios since we are filtering out approximately the same extended emission in all transitions and therefore we are obtaining information from the same spatial scales. This is also evidenced by the similar line widths from all of our observed line profiles (e.g., in Fig. 2). As mentioned above and followed up in Sect. 4.2, this issue poses some questions regarding the accuracy and interpretation of results obtained from low resolution and non-simultaneous heterogeneous observations.

Figure 2 also shows that should ${ }^{13} \mathrm{C}^{18} \mathrm{O}$ be affected by a similar flux filtering as the other isotopologues, assuming a correct baseline subtraction, it should have potentially been detected at higher signal to noise ratio in the IRAM $30 \mathrm{~m}$ single dish data by both Martín et al. (2010) and Aladro et al. (2015).

\subsubsection{Line ratio maps}

In Fig. 3 we show the integrated flux density ratio maps for the relevant pairs of transitions. These ratios have been calculated for pixels where the signal in the integrated maps in the $50-450 \mathrm{~km} \mathrm{~s}^{-1}$ range is $>1 \sigma$, which is $>30 \mathrm{mJy}_{\text {beam }}^{-1} \mathrm{~km} \mathrm{~s}^{-1}$ in ${ }^{13} \mathrm{CO}, \mathrm{C}^{18} \mathrm{O}$, and $\mathrm{C}^{17} \mathrm{O}$, and $>9.5 \mathrm{mJybeam}^{-1} \mathrm{~km} \mathrm{~s}^{-1}$ for ${ }^{13} \mathrm{C}^{18} \mathrm{O}$. The uncertainty in these ratio maps is described in Appendix A. The first central $\mathrm{C}^{18} \mathrm{O}$ contour fulfilling that criterion is shown in both Figs. 3 and A.1, and is considered to be the region of relevance for these ratios. Outside this area, although there is emission above the thresholds, we cannot ensure these are not residuals from the cleaning process.

The average and standard deviation within this region with different weighting schemes are shown in Table 1 for each line ratio. The colour scale in Fig. 3 was set from 0 to the unweighted average plus twice the standard deviation. On such a colour scale, the $\mathrm{C}^{18} \mathrm{O} /{ }^{13} \mathrm{C}^{18} \mathrm{O}$ ratios appear to be very similar to those of ${ }^{13} \mathrm{CO} /{ }^{13} \mathrm{C}^{18} \mathrm{O}$ (upper two panels of Fig. 3), indicating a rather constant ${ }^{13} \mathrm{CO} / \mathrm{C}^{18} \mathrm{O}$ line intensity ratio as can also be seen in the lowest panel of Fig. 3 . The $\mathrm{C}^{18} \mathrm{O} /{ }^{13} \mathrm{C}^{18} \mathrm{O}$ and ${ }^{13} \mathrm{CO} /{ }^{13} \mathrm{C}^{18} \mathrm{O}$ ratios appear to show a gradient from the inner region towards the outskirts of the CMZ where some clumps show very high ratios. We note that the decrease of the ratio towards the centre (bluish regions in the upper two panels of Fig. 3) is caused by the increased line contamination in ${ }^{13} \mathrm{C}^{18} \mathrm{O}$ as seen in Fig. 1. Thus, $\mathrm{C}^{18} \mathrm{O} /{ }^{13} \mathrm{C}^{18} \mathrm{O}$ and ${ }^{13} \mathrm{CO} /{ }^{13} \mathrm{C}^{18} \mathrm{O}$ ratio maps as well as their derived averages in Table 1 need to be considered with caution in this region as discussed in Sect. 4.1. $\mathrm{C}^{18} \mathrm{O} / \mathrm{C}^{17} \mathrm{O}$ and ${ }^{13} \mathrm{CO} / \mathrm{C}^{18} \mathrm{O}$ appear somewhat smoother, and indeed their relative dispersion in Table 1 are lower than in the other ratios.

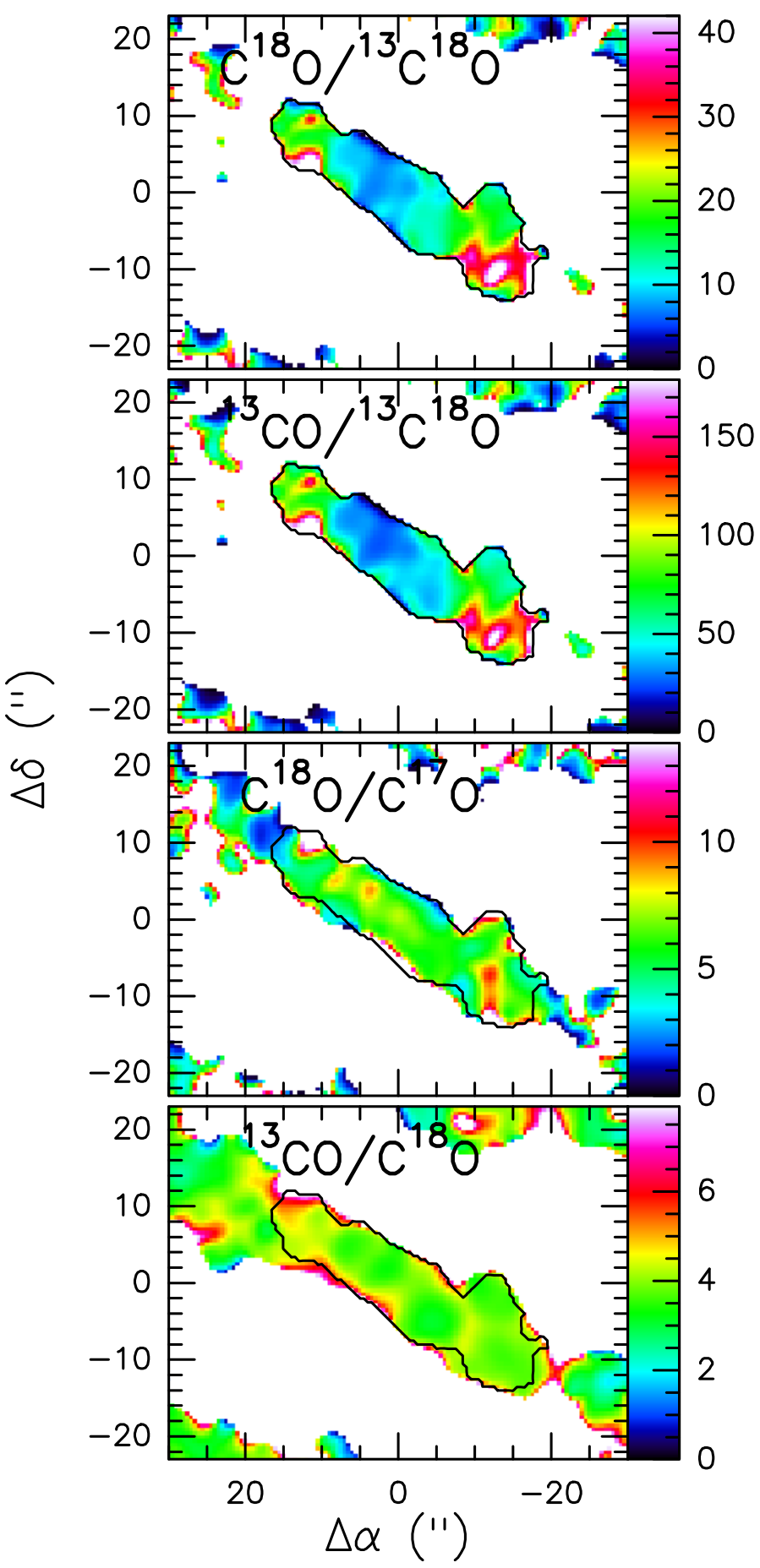

Fig. 3. Integrated flux density ratio maps derived from the maps shown in Fig. 1. Only the ratios discussed in the paper are shown. The black contour is the central $1 \sigma$ level of $\mathrm{C}^{18} \mathrm{O}$, used to derive ratio averages within the maps. See Sect. 3.1.2 for details.

\subsection{Spectral analysis at selected positions}

The positions studied in this work were selected by visually inspecting all the velocity channels in the $\mathrm{C}^{18} \mathrm{O}$ data cube where individual flux density maxima were identified. The coordinates of the positions identified are listed in Table $2 . \mathrm{C}^{18} \mathrm{O}$ was selected for this task because it is bright while not affected by the effects of large optical depths, potentially affecting the brighter ${ }^{13} \mathrm{CO}$, across the whole CMZ. The velocity structure and peak positions are similar for all isotopologues so the position selection is 
Table 1. Averaged derived isotopic ratios in NGC 253.

\begin{tabular}{|c|c|c|c|c|c|c|c|}
\hline \multirow{2}{*}{$\begin{array}{c}\text { Isotopic } \\
\text { ratio }\end{array}$} & \multirow{2}{*}{$\begin{array}{l}\text { Molecular } \\
\text { ratio }\end{array}$} & \multicolumn{3}{|c|}{ Maps ${ }^{(a)}$} & \multicolumn{3}{|c|}{ Spectra $^{(b)}$} \\
\hline & & unweighted & $\sigma$-weighted & snr-weighted & unweighted & $\sigma$-weighted & $\tau$-weighted \\
\hline${ }^{12} \mathrm{C} /{ }^{13} \mathrm{C}$ & $\mathrm{C}^{18} \mathrm{O} /{ }^{13} \mathrm{C}^{18} \mathrm{O}$ & $18 \pm 12$ & $13 \pm 8$ & $11 \pm 5$ & $22 \pm 7$ & $21 \pm 6$ & $23 \pm 5$ \\
\hline${ }^{16} \mathrm{O} /{ }^{18} \mathrm{O}$ & ${ }^{13} \mathrm{CO} /{ }^{13} \mathrm{C}^{18} \mathrm{O}$ & $75 \pm 52$ & $48 \pm 30$ & $40 \pm 20$ & $140 \pm 50$ & $130 \pm 40$ & $140 \pm 40$ \\
\hline${ }^{18} \mathrm{O} /{ }^{17} \mathrm{O}$ & $\mathrm{C}^{18} \mathrm{O} / \mathrm{C}^{17} \mathrm{O}$ & $6.5 \pm 3.7$ & $6.2 \pm 1.6$ & $5.7 \pm 1.6$ & $4.6 \pm 1.1$ & $4.5 \pm 0.8$ & $4.7 \pm 0.8$ \\
\hline & ${ }^{13} \mathrm{CO} / \mathrm{C}^{18} \mathrm{O}$ & $4.5 \pm 1.7$ & $3.8 \pm 0.6$ & $3.8 \pm 0.5$ & $6.2 \pm 1.0$ & $6.1 \pm 0.9$ & $6.1 \pm 0.9$ \\
\hline
\end{tabular}

Notes. ${ }^{(a)}$ Averages and standard deviations derived from flux density ratio maps calculated within the region of interest in Fig. 3. See Sect. 3.1.2 for details. Parameters have been calculated unweighted, as well as weighted by the $\sigma$ and signal to noise in each pixel (Appendix A). ${ }^{(b)}$ Averages and standard deviations of the mean derived from the ratios of the column densities in Table 2, measured from the extracted spectra at the positions indicated in Fig. 1. Positions with upper limits are not considered. Similarly to the maps, parameters have been calculated unweighted, weighted with the standard deviation of each measurement, and weighted with the optical depth of the ${ }^{13} \mathrm{CO}$ transition in Table 2 . See Sect. 4.1 for a discussion on the differences observed between the maps and the spectra.

not biased towards ${ }^{18} \mathrm{O}$ enhanced regions. This selection was not based on the previously identified GMC locations from existing high resolution studies of multi-molecular studies of NGC 253 (see Appendix B) since the purpose of this selection was to identify the maxima in our maps to enhance the detectability of the fainter isotopologues .

The integrated map of ${ }^{13} \mathrm{CO}$ in Fig. 1 shows the location of these positions. Table 2 also lists the projected galactocentric distance of each position referred to the bright compact radio source at $\alpha_{\mathrm{J} 2000}=00^{\mathrm{h}} 47^{\mathrm{m}} 33^{\mathrm{s}} \cdot 17, \delta_{\mathrm{J} 2000}=-25^{\circ} 17^{\prime} 17^{\prime \prime} \cdot 1$ (Turner \& Ho 1985; Ulvestad \& Antonucci 1997) assuming a distance of $3.5 \mathrm{Mpc}$ to NGC 253. This distance is projected on the plane of the sky (i.e. not corrected for inclination) and may differ from the actual galactocentric distance, also among velocity components within a given position. Some pairs of positions are very close in projected distance (i.e. positions 6 and 7) but they correspond to the maximum flux densities of two distinct velocity components identified around those positions.

\subsection{1. ${ }^{13} \mathrm{C}^{18} \mathrm{O}$ line contamination}

Martín et al. (2010) identified the contribution of the $\mathrm{H}_{2} \mathrm{CS}$ $3_{1,2}-2_{1,1}$ transition at $104.616 \mathrm{GHz}$ to the ${ }^{13} \mathrm{C}^{18} \mathrm{O}$ line profile. The detection of $\mathrm{H}_{2} \mathrm{CS}$ is actually confirmed by the detection within our observed bands of the $3_{0,3}-2_{0,2}$ line at $103.040 \mathrm{GHz}$ with the expected relative flux density.

However, in our high sensitivity data, we also identify two closer features to the ${ }^{13} \mathrm{C}^{18} \mathrm{O}(1-0)$ transition, both emitting at lower frequencies (higher velocities). This is particularly obvious in the positions 6 and 7 in Table 2 as shown in Fig. 4 for position 6. As seen in that Figure, the observed profile is not similar to that of ${ }^{13} \mathrm{CO}$, shown at a scaled down intensity for reference. The line shapes of $\mathrm{C}^{17} \mathrm{O}$ and $\mathrm{C}^{18} \mathrm{O}$, however, match that of ${ }^{13} \mathrm{CO}$. We note that these two positions are the most affected by this contamination.

In order to identify the origin of the emission blended to ${ }^{13} \mathrm{C}^{18} \mathrm{O}$ we performed a preliminary fit to the spectra in these two positions using four Gaussian profiles; we note that the final fit in Fig. 4 include more Gaussian profiles to account for the faint velocity components of the contaminants. The velocity of the first two were fixed to those of the two components observed in ${ }^{13} \mathrm{CO}$, to account for the emission of ${ }^{13} \mathrm{C}^{18} \mathrm{O}$. The other two, accounting for the contaminant emission other than the already identified $\mathrm{H}_{2} \mathrm{CS}$ line, had the velocity as free parameter but the width was fixed to that of the brighter ${ }^{13} \mathrm{CO}$ component. The features which are blended to ${ }^{13} \mathrm{C}^{18} \mathrm{O}$ appear at $+23.0 \pm 1.3 \mathrm{~km} \mathrm{~s}^{-1}$, and $+129.2 \pm 1.4 \mathrm{~km} \mathrm{~s}^{-1}$ with respect to the velocity of the brightest component of ${ }^{13} \mathrm{C}^{18} \mathrm{O}$, which sets the rest frequencies of the contaminating emission at $104703.3 \pm 0.5 \mathrm{MHz}$, and $104666.3 \pm 0.5 \mathrm{MHz}$, respectively.

The spectral scans towards Sgr B2 and the Orion hot cores by Turner (1989) indeed show an unidentified feature at $104696 \mathrm{MHz}$ but nothing at lower frequencies, while the scan by Belloche et al. (2013) towards Sgr B2(N) shows transitions of $\mathrm{CH}_{3} \mathrm{OCH}_{3}$ and $\mathrm{c}-\mathrm{C}_{2} \mathrm{H}_{4} \mathrm{O}$ around the frequencies of interest, overlapped with a number of vibrational transitions of $\mathrm{C}_{2} \mathrm{H}_{3} \mathrm{CN}$. Towards Sgr B2(M), on the other hand, Belloche et al. (2013) detected just faint $\mathrm{CH}_{3} \mathrm{OCH}_{3}$ emission around $104.7 \mathrm{GHz}$. However, based on our LTE synthetic spectrum of $\mathrm{CH}_{3} \mathrm{OCH}_{3}$, a number of other transitions of this species should have been detected within our observed bandwidth, but they are not, which implies that $\mathrm{CH}_{3} \mathrm{OCH}_{3}$ does not contribute significantly to the observed profile. By using the spectroscopic information in the JPL and CDMS catalogues (Pickett et al. 1998; Müller et al. 2001, 2005) we identified that our estimated frequencies agree well with those of the hyperfine transitions of $\mathrm{C}_{4} \mathrm{H}$ at $104705 \mathrm{MHz}$ $\left(11_{11}-10_{10}\right)$ and $104666 \mathrm{MHz}\left(11_{12}-10_{11}\right)$ with upper level energies $E_{\mathrm{u}}=30 \mathrm{~K}$. Just recently, $\mathrm{C}_{4} \mathrm{H}$ was detected for the first time in NGC 253 by Mangum et al. (2019) who reported higher energy transitions $\left(E_{\mathrm{u}}=126 \mathrm{~K}\right)$ in higher resolution observations. These are the first extragalactic detections of $\mathrm{C}_{4} \mathrm{H}$ in emission, only previously reported towards the absorption system PKS 1830-211 (Muller et al. 2011).

Despite the strong blending between ${ }^{13} \mathrm{C}^{18} \mathrm{O}$ and the $11_{11}-10_{10}$ transitions of $\mathrm{C}_{4} \mathrm{H}$ (low velocity $\mathrm{C}_{4} \mathrm{H}$ transition in Fig. 4), we were able to account for the contribution of this transition to the observed spectral feature thanks to the relatively cleaner $\mathrm{C}_{4} \mathrm{H} 11_{12}-10_{11}$ transitions (higher velocity $\mathrm{C}_{4} \mathrm{H}$ transition in Fig. 4). So, when analysing the spectra (Sect. 3.2.2), we considered as many velocity components of $\mathrm{C}_{4} \mathrm{H}$ as those fitted to ${ }^{13} \mathrm{C}^{18} \mathrm{O}$. To de-blend the emission of ${ }^{13} \mathrm{C}^{18} \mathrm{O}$ from that of $\mathrm{C}_{4} \mathrm{H}$ we proceeded as follows. We fitted simultaneously the velocity components of ${ }^{13} \mathrm{C}^{18} \mathrm{O}$ and $\mathrm{C}_{4} \mathrm{H}$. For the latter we used only the $11_{12}-10_{11}$ transitions (farther from the ${ }^{13} \mathrm{C}^{18} \mathrm{O}$ transition and therefore cleaner) to fit to the observed $\mathrm{C}_{4} \mathrm{H}$ emission. Based on the fit to the $11_{12}-10_{11}$ transitions, the contribution of the $11_{11}-10_{10}$ lines (strongly blended with ${ }^{13} \mathrm{C}^{18} \mathrm{O}$ ) was then estimated from the expected relative intensities calculated with the spectroscopic parameters of the $\mathrm{C}_{4} \mathrm{H}$ transitions involved. ${ }^{13} \mathrm{C}^{18} \mathrm{O}$ emission was then fitted after removing the contribution of $\mathrm{C}_{4} \mathrm{H}$. In some cases where the fainter velocity components of ${ }^{13} \mathrm{C}^{18} \mathrm{O}$ were partially blended to the $11_{12}-10_{11} \mathrm{C}_{4} \mathrm{H}$ 


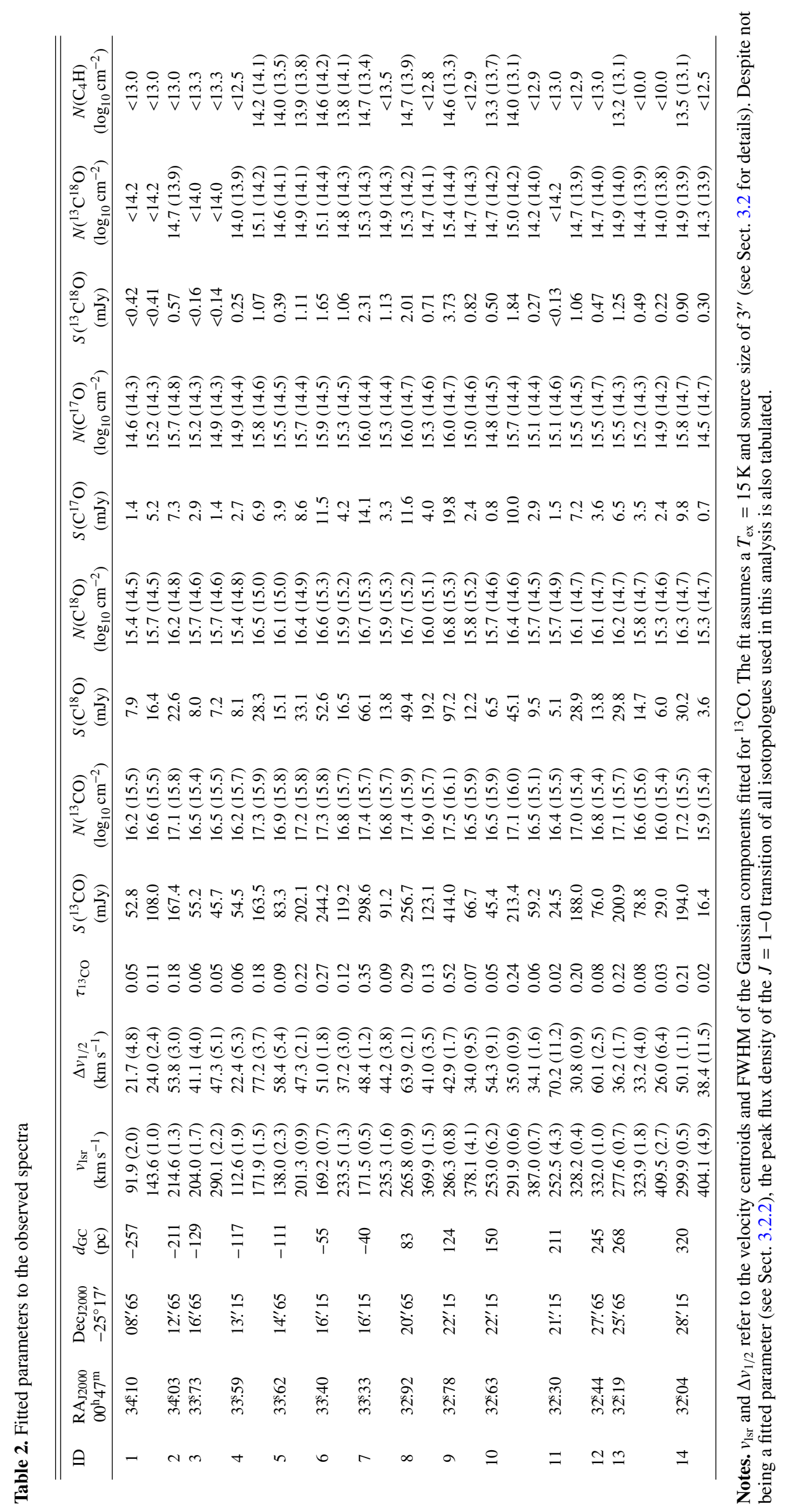




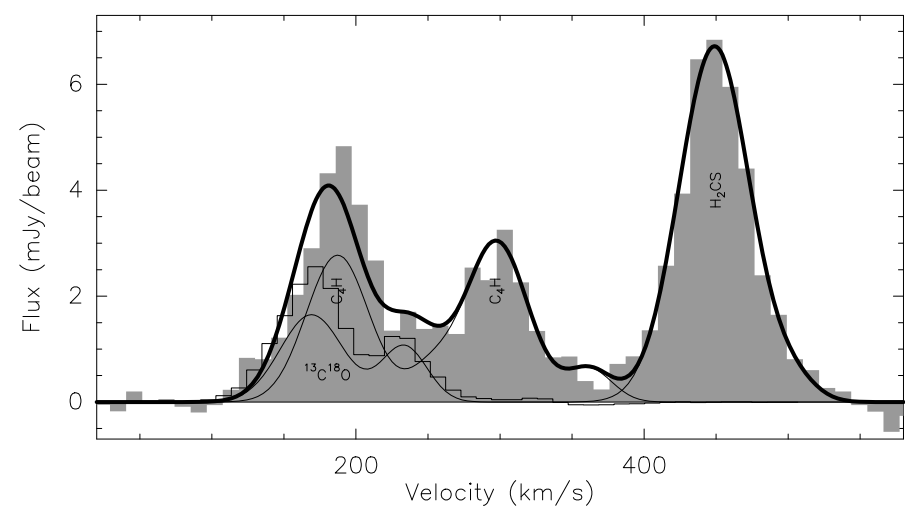

Fig. 4. Spectra extracted at position 6 in Table 2. The grey filled histogram shows the spectrum around the ${ }^{13} \mathrm{C}^{18} \mathrm{O}$ transition and the velocity scale refers to its rest frequency. The thick curve shows the overall fitted profile to the observations, while the thinner curves show the profiles of ${ }^{13} \mathrm{C}^{18} \mathrm{O}$, as well as the identified emission of the hyperfine structure of $\mathrm{C}_{4} \mathrm{H}$ (see Sect. 3.2.1 for details) and the line of $\mathrm{H}_{2} \mathrm{CS}$. The empty histogram shows the ${ }^{13} \mathrm{CO}$ profile (divided by 100 ) at the same position as a reference.

transitions, this fitting procedure required to be iterated until the fit to all components converged. We also note that the fainter velocity components are subject to stronger blending uncertainties as illustrated in Fig. 4, resulting in large errors in the fits of both $\mathrm{C}_{4} \mathrm{H}$ and ${ }^{13} \mathrm{C}^{18} \mathrm{O}$. However, the values in Table 2 can be considered as clean ${ }^{13} \mathrm{C}^{18} \mathrm{O}$ column densities compared to the ${ }^{13} \mathrm{C}^{18} \mathrm{O}$ integrated intensity map in Fig. 1 , where the $\mathrm{C}_{4} \mathrm{H}$ emission is not removed (Sect. 4.1).

\subsection{2. ${ }^{13} \mathrm{C}^{18} \mathrm{O}$ LTE analysis}

Spectra were extracted at each of the selected positions and modelled under the local thermodynamic equilibrium (LTE) assumption using MADCUBA ${ }^{2}$ (Martín et al., in prep.). With MADCUBA we fit a synthetic spectrum, calculated from input physical parameters and using the molecular spectroscopic parameters in the JPL and CDMS catalogues, to the observed line profiles. The free input parameters that can be fitted are the column density, excitation temperature, velocity, line width, and source size of each molecular species. Figure 4 shows a sample of the synthetic combined profile overlaid on top of the observed spectrum in one of the positions in this study. The results of the multi-Gaussian-component modelling to all selected positions are shown in Table 2 where the obtained column densities derived for each isotopologue, as well as for $\mathrm{C}_{4} \mathrm{H}$, are given. The peak flux densities of the observed $\mathrm{CO}$ isotopologues spectral lines, even though it is not a direct fitted parameter since MADCUBA does fit the column density, are also listed in Table 2 for reference.

Since this study is based on a single transition of each $\mathrm{CO}$ isotopologue, we need to make a number of assumptions: $i$ ) all isotopologues have the same distribution, including velocity profile; $i$ ) they have a common excitation temperature of $T_{\mathrm{ex}}=15 \mathrm{~K}$; and iii) the source size is $3^{\prime \prime}$, matching the map resolution. We explain the implications of such assumptions below.

The velocities and widths were fitted to the ${ }^{13} \mathrm{CO}$ profiles, and then set as fixed parameters when fitting the other isotopologues and $\mathrm{C}_{4} \mathrm{H}$. This constraint was imposed to ensure consistency in the line profiles fitted, but in any case, letting these

2 http://cab.inta-csic.es/madcuba/Portada.html parameters free resulted in consistent values within the errors in the cases where the fit was possible both in terms of having enough signal to noise and not being significantly affected by line blending. Thus, fixing the velocity and width was only critical in the spectra with low signal to noise ratios and those of ${ }^{13} \mathrm{C}^{18} \mathrm{O}$ where line blending was observed (Sect. 3.2.1).

The excitation temperature was fixed to $15 \mathrm{~K}$ based on the rotational temperatures derived from multi-transition studies based on previous spectral scans (Martín et al. 2006) and on preliminary results from the ALMA multi-band spectral scan on NGC 253 (Martín et al., in prep.). Differences in the assumed excitation temperature have a minor impact on the derived column densities. For temperatures of 10 and $20 \mathrm{~K}$, we estimate column densities $\sim 5 \%$ and $\sim 10 \%$ higher, respectively. However this would affect all isotopologues similarly if we assume they share similar excitation conditions, and therefore column density ratios would remain virtually unchanged. For temperatures below $10 \mathrm{~K}$ it is not possible to fit the intensity of observed profiles for any combination of column density and source size.

The column density and optical depth of the ${ }^{13} \mathrm{CO}$ transition are directly linked to the assumed source size, therefore these values would both increase under the assumption of a smaller source size. High resolution imaging by Ando et al. (2017) resulted in resolved GMCs of $\sim 9 \mathrm{pc}\left(0.5^{\prime \prime}\right)$ in size. However, in the case of position 9 in Table 2, the position with the largest measured optical depth (Table 2), we are not able to reproduce the observed flux density for source sizes $<2$ " unless significantly increasing the excitation temperature. However as indicated above, multi-transition studies do not point towards such high excitation temperatures. Thus we assume that the observed profiles stem from $2-3^{\prime \prime}$ averaged regions. For a source size of $2^{\prime \prime}$, derived ${ }^{13} \mathrm{CO}$ column densities in Table 2 would be underestimated by up to a factor of 3 in the brightest position 9 . However, for all other positions, less affected by optical thickness, ${ }^{13} \mathrm{CO}$ column densities would be underestimated by a factor of $\lesssim 2.3$. On the other hand, the column density ratios in this study, would be underestimated by up to $40 \%$ in position 9 for the ratios involving ${ }^{13} \mathrm{CO}$, but we enter into the optically thick regime where fitted parameters strongly depend on the assumptions of source size and excitation temperature. For the optically thinner lines of sights and isotopologues, the derived ratios would mostly be unaltered.

Based on the column densities in Table 2, in Table 1 we show the average of the column density ratios and standard deviations calculated with the values obtained from all selected positions. These ratios do not include the positions where only upper limits to the ${ }^{13} \mathrm{C}^{18} \mathrm{O}$ were obtained. The averages and standard deviations are calculated: unweighted as a raw value, weighted by the standard deviation of individual measurements to get the average of the ratios measured at higher signal to noise or less blending, and weighted by the optical depth of ${ }^{13} \mathrm{CO}$ as representative of the most massive clouds sampled.

\section{Discussion}

\subsection{Map versus spectra ratios: Optical depth and contamination effects.}

From Table 1 we see how the average isotopologue ratios, calculated as the column density ratios derived from the spectral analysis (Sect. 3.2.2) vary little and within the uncertainties when different weighting schemes are used. Although still consistent within the error bars (when unweighted averages are considered), these values from spectral analysis are higher than those 

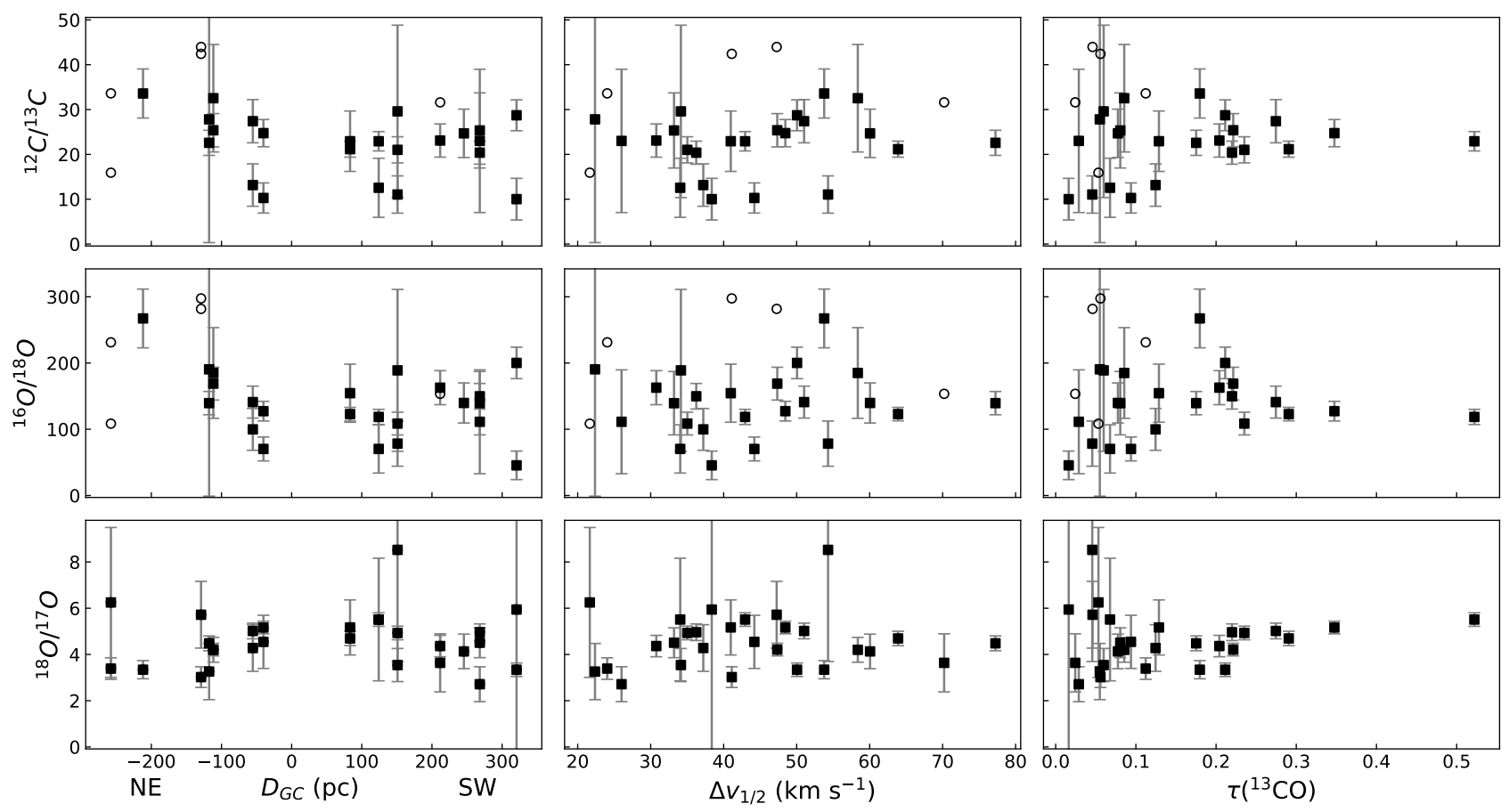

Fig. 5. Measured isotopic ratios as a function of galactocentric distance, FWHM of the fitted profile, and optical depth of the ${ }^{13} \mathrm{CO}$ transition. Ratios are calculated based on the column density ratios in Table 2. Lower limits $(3 \sigma)$ to the ratios due to non-detection of ${ }^{13} \mathrm{C}^{18} \mathrm{O}$ are represented as open circles.

measured from the averaging of the maps. These differences can be considered significant since they are both measured with the same dataset. We discuss why these difference can be attributed to a combined effect of both optical depth and line contamination affecting the lines in the measured ratio.

As explained above, ${ }^{13} \mathrm{CO}$ is affected by significant optical depth (Sect. 3.2.2) while ${ }^{13} \mathrm{C}^{18} \mathrm{O}$ is contaminated by $\mathrm{C}_{4} \mathrm{H}$. Thus, we observe that the optical depth affected ratio ${ }^{13} \mathrm{CO} / \mathrm{C}^{18} \mathrm{O}$ is $38 \pm 6 \%$ smaller in the averaged map than from the spectra (considering unweighted values from Table 1). On the other hand, the contamination affected ratio $\mathrm{C}^{18} \mathrm{O} /{ }^{13} \mathrm{C}^{18} \mathrm{O}$ is observed to be $22 \pm 9 \%$ smaller. Subsequently, the ${ }^{13} \mathrm{CO} /{ }^{13} \mathrm{C}^{18} \mathrm{O}$ ratio, affected by both effects, should differ by approximately the multiplication of both factors, and therefore be $70 \pm 40 \%$ smaller and, indeed the observed difference between the spectra and map derived average is $90 \pm 40 \%$.

This explanation is further supported by the fact that the derived averages diverge even further from those derived with the spectra when weighted by the standard deviation or the signal to noise ratio per pixel. These weightings favour the brighter regions, which are those that are either more opaque and/or more contaminated by $\mathrm{C}_{4} \mathrm{H}$.

On the other hand, the $\mathrm{C}^{18} \mathrm{O} / \mathrm{C}^{17} \mathrm{O}$ ratio is not expected to be affected by line saturation and we observe that this is the only ratio which is higher in the averaged maps compared to the spectra and which show little variation with the weighted averages from the maps. Therefore, the differences between the values derived from the maps and the spectra can be actually attributed to actual spatial variations. We note that the small variation derived from the weighted average in the $\mathrm{C}^{18} \mathrm{O} / \mathrm{C}^{17} \mathrm{O}$ ratio, could still be due to significant optical depth in the $\mathrm{C}^{18} \mathrm{O}$ emission.

Based on the arguments above we can safely consider that the values derived from the spectra are the most accurate optical depth and contamination corrected values as proxies of the elemental isotopic ratios in NGC 253. While we adopt the $\sigma$ weighted ratios from the spectra for the discussion on stellar processing in Sect. 4.4, we use the unweighted values as reference for comparison with previous single dish data in the literature in Sect. 4.2.

\subsection{Spatially resolved carbon and oxygen ratios with optically thin tracers}

Based on the observations presented in this paper, we can for the first time derive spatially resolved carbon and oxygen isotopic ratios based on the rarer carbon monoxide isotopologues. This is under the assumption that the column density ratio of the molecular isotopologues reflects the actual atomic isotopic ratio.

As seen in Figs. 3 and 5, the measured isotopic ratios vary significantly across the CMZ in NGC 253. Based on the column density values determined from the spectra (Table 2), we observe ratios ranging from 10 to 34 for ${ }^{12} \mathrm{C} /{ }^{13} \mathrm{C}, 45$ to 270 for ${ }^{16} \mathrm{O} /{ }^{18} \mathrm{O}$, and to a lesser extent 2.7 to 8.5 for ${ }^{18} \mathrm{O} /{ }^{17} \mathrm{O}$. Still, on average our high resolution results are about a factor of two below those typically assumed in the nuclei of galaxies (Wilson \& Rood 1994; Henkel et al. 1994; Wang et al. 2004).

As indicated in the introduction the selection of optically thin tracers minimizes the effect of selective photodissociation. However, if ${ }^{13} \mathrm{C}^{18} \mathrm{O}$ should be affected by a higher photodissociation rate (Visser et al. 2009), the ratios derived in this paper would be even lower. However, we have no evidence to support such effect globally within the central region of NGC 253.

We put our results in the context of previously determined isotopic ratios towards NGC 253.

\subsection{1. ${ }^{12} \mathrm{C} /{ }^{13} \mathrm{C}$}

The single dish ratio $\mathrm{C}^{18} \mathrm{O} /{ }^{13} \mathrm{C}^{18} \mathrm{O} \gtrsim 60$ reported by Martín et al. (2010) differs by a factor of 3 from the ratio reported in 
this work. We note that the $\mathrm{C}^{18} \mathrm{O}$ peak temperature reported by Martín et al. (2010) is $75 \%$ brighter than that reported by Aladro et al. (2015). Additionally, ${ }^{13} \mathrm{C}^{18} \mathrm{O}$ and $\mathrm{C}^{18} \mathrm{O}$ were observed at different positions, SD B and SD A, respectively. As explained in Sect. $3.1 .1,{ }^{13} \mathrm{C}^{18} \mathrm{O}$ could be $25 \%$ brighter towards the SD A position. Thus, if these uncertainties align in the same direction, the ratio derived by Martín et al. (2010) could result in a value as low as $\gtrsim 35$, which is closer to the value derived with other tracers (Henkel et al. 2014), but still higher than the value derived in this paper. However, the single dish value from Martín et al. (2010) is uncertain because of the unknown systematic errors resulting from the use of heterogeneous datasets.

The value derived from our optically thin observations is a factor of 2 lower than the ratio typically assumed for galaxies (Wilson \& Rood 1994; Henkel et al. 2014) and a factor of 2-4 below the limits based on CCH (Martín et al. 2010), in this case derived from the homogeneous spectral scan dataset by Martín et al. (2006).

As we discuss in Sect. 4.4, this difference may be real and a result of spatially distinct molecular gas component with different degrees of stellar processing.

\subsection{2. ${ }^{16} \mathrm{O} /{ }^{18} \mathrm{O}$}

The value quoted in the literature (Wilson \& Rood 1994) of $\sim 200$ is derived from the ${ }^{13} \mathrm{CO} / \mathrm{C}^{18} \mathrm{O}$ ratio reported by Sage et al. (1991) in a sample of three galaxies (NGC 253, IC 342, M 82), and multiplied by the assumed ${ }^{12} \mathrm{C} /{ }^{13} \mathrm{C} \sim 40$ (Henkel et al. 1993). For NGC 253, Sage et al. (1991) reported ${ }^{13} \mathrm{CO} / \mathrm{C}^{18} \mathrm{O}=$ $4.9 \pm 0.5$, which is good agreement with the value of $4.5 \pm 1.7$ derived from our map measurements in Table 2. If we use our derived ${ }^{12} \mathrm{C} /{ }^{13} \mathrm{C}$ to multiply the measurement by Sage et al. (1991), we obtain a ratio ${ }^{16} \mathrm{O} /{ }^{18} \mathrm{O}=110 \pm 30$, which is consistent with our optical depth/contamination corrected (Sect. 3.2) measurement of ${ }^{13} \mathrm{CO} /{ }^{13} \mathrm{C}^{18} \mathrm{O}$. Thus, the discrepancy between the ${ }^{16} \mathrm{O} /{ }^{18} \mathrm{O}$ ratio reported in this work and that of Wilson \& Rood (1994) resides on the large scale ${ }^{12} \mathrm{C} /{ }^{13} \mathrm{C}$ ratio differing from that measured at high resolution.

\subsection{3. ${ }^{18} \mathrm{O} /{ }^{17} \mathrm{O}$}

Sage et al. (1991) reported a ${ }^{18} \mathrm{O} /{ }^{17} \mathrm{O}$ of $10 \pm 2.5$ based on $\mathrm{C}^{18} \mathrm{O} / \mathrm{C}^{17} \mathrm{O} J=2-1$ observations towards NGC 253 . The discrepancy can be attributed to the low signal to noise ratio of their single dish data. The higher sensitivity observations by Aladro et al. (2015) of the $J=1-0$ yield a ratio of $7.1 \pm 0.4$, also in good agreement with the map derived value of $6.5 \pm 3.7$ in Table 1.

\subsection{Isotopic ratio variations across the $C M Z$ of NGC 253}

Since the ALMA observations allowed us to spatially resolve the CMZ of NGC 253 we explore the variations of isotopic ratios among the individual identified sources across this region.

Within our Galaxy, a clear gradient as a function of the galactocentric distance is observed in the carbon, oxygen and nitrogen isotopic ratios (Gardner \& Whiteoak 1979; Henkel et al. 1982; Langer \& Penzias 1990; Wilson \& Rood 1994; Goto et al. 2003; Milam et al. 2005; Wouterloot et al. 2008; Zhang et al. 2015). The left panels in Fig. 5 show the variation of estimated isotopic ratios as a function of the linear projected distance measured from the assumed galactic centre position (see Sect. 3.2). We do not observe an obvious gradient in our data as a function of galactocentric distance. However, we note that, within the Galaxy, this gradient is observed at distances ranging 3-10 kpc, while our observations in NGC 253 cover the inner $\sim 300 \mathrm{pc}$. In fact, considering the dispersion of the observations at a given Galactocentric distance, such gradient, should it continue towards the very central region, might not be distinguished within the central few hundred parsecs of the Galaxy as is the case in NGC 253. Most of these studies present only one observational point towards the Galactic centre ranging ${ }^{12} \mathrm{C} /{ }^{13} \mathrm{C}=17 \pm 7$ (where the error includes the variation of the ratio measured with different molecular tracers used, Langer \& Penzias 1990; Milam et al. 2005) with a standard value generally adopted of 20 (Wilson \& Rood 1994). Moreover, the study by Gardner \& Whiteoak (1982) shows that the low ratio of ratio ${ }^{12} \mathrm{C} /{ }^{13} \mathrm{C} \sim 15 \pm 4$, as measured with $\mathrm{H}_{2} \mathrm{CO}$, is relatively homogeneously observed across the $\mathrm{CMZ}$ and not exclusively from individual sources like Sgr A or Sgr B2. This is similar to the relatively homogeneous ratios observed within the CMZ of NGC 253. Similarly, oxygen ratios show no variations with distance from the centre of NGC 253 within a radius of 300 pc. Similarly, we do not observe obvious gradients in ${ }^{16} \mathrm{O} /{ }^{18} \mathrm{O}$ and ${ }^{18} \mathrm{O} /{ }^{17} \mathrm{O}$ ratios, with observed ratios within $25 \%$ of the average (see Table 1 ).

In order to further investigate the relative variations of the isotopic ratios among molecular components, we plot the measured ratios as a function of the observed line width. Line width was taken as a proxy of the virial mass of the unresolved clouds because of the lack of an a priori knowledge of their individual sizes. Then central panels in Fig. 5 show no apparent trend with the line width on any of the isotopic ratios.

The right panels in Fig. 5 show the ratio dependency on the measured optical depth of ${ }^{13} \mathrm{CO}\left({ }^{13} \tau\right)$ at each position. This optical depth assumes the same source size for all sources. The implications of this assumption have already been discussed in Sect. 3.2 and translate into an uncertainty in the $x$-axis of this dependency, where points might be displaced by up to a factor of $\sim 2$ towards higher optical depths for some positions. Once again, no obvious trend is observed. However the most uncertain ratios are found towards the weaker molecular components such as those with lower optical depths and therefore column densities; this results in larger error bars and uncertain lower limits in the ${ }^{12} \mathrm{C} /{ }^{13} \mathrm{C}$ and ${ }^{16} \mathrm{O} /{ }^{18} \mathrm{O}$ owing to the strong blending with $\mathrm{C}_{4} \mathrm{H}$ (Sect. 3.2.1). This is also reflected in larger uncertainties due to the limited signal to noise ratios in ${ }^{18} \mathrm{O} /{ }^{17} \mathrm{O}$, unaffected by blending.

Additionally, in Fig. 6 we show the variations of the carbon and oxygen isotopic ratios across all the positions selected, where the different velocity components are separated into four colour groups, according to the measured ratios, where also the optical depth of ${ }^{13} \mathrm{CO}$ is coded with the size of the points. Higher granularity in the colour coding does not provide a more accurate picture given the errors in the ratios.

In contrast to the situation in our Galactic centre (Fig. 2 in Gardner \& Whiteoak 1982), in this work we can assume that all velocity components are located within the CMZ. However our spatial resolution is not high enough to provide an accurate picture of their locations. Higher resolution observations will provide a more accurate picture of the isotopic distribution.

We observe an overall homogeneity of the measured isotopic ratios where most of the gas is around the averaged reported values. Only a few low column density velocity components show ratios significantly above or below the average (blue and red, respectively, in Fig. 6). 

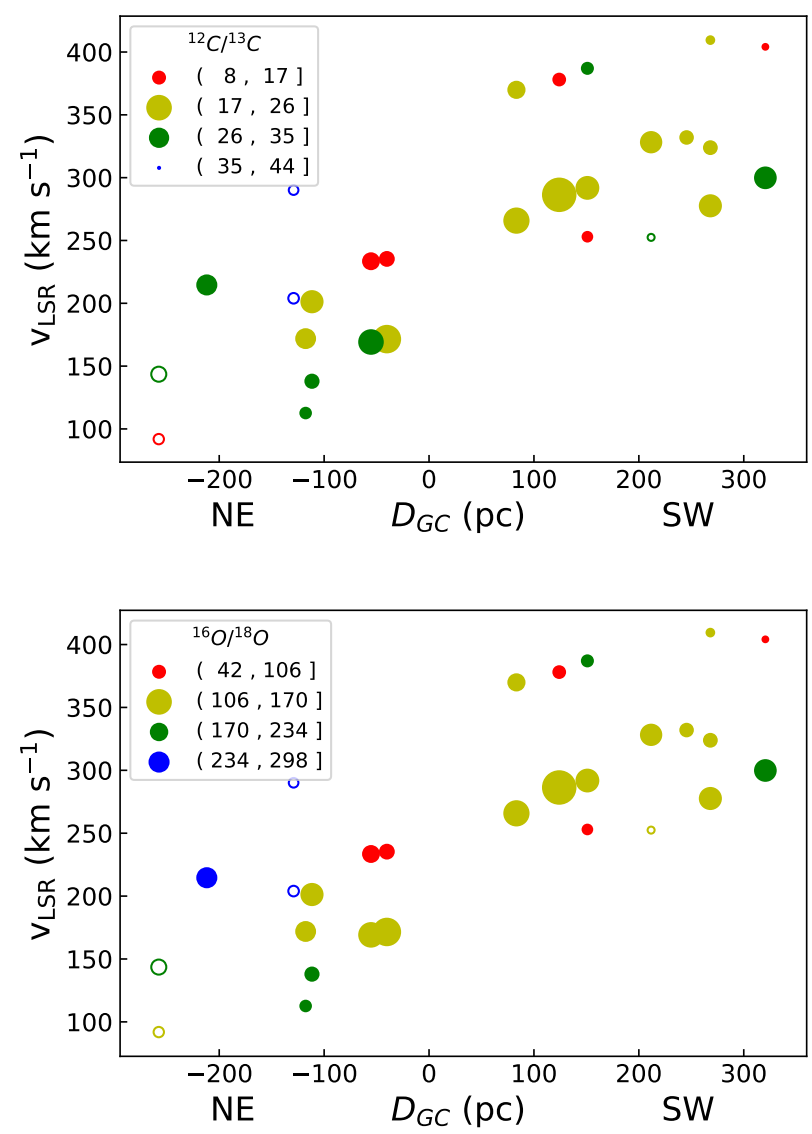

Fig. 6. Colour-coded carbon (top panel) and oxygen (bottom panel) isotopic ratios as a function of the distance to the galaxy centre and separated by the measured velocity of each fitted component. Colourcoding ranges were selected to distinguish four quartiles between the minimum and maximum values measured in all components. The size of the points is related to the optical depth of the ${ }^{13} \mathrm{CO}$. Open symbols represent lower limits.

\subsection{Stellar processed gas in the galactic centre of NGC 253}

The relatively large ${ }^{12} \mathrm{C} /{ }^{13} \mathrm{C}$ ratio observed in NGC 253 (and other starbursts galaxies) as compared to that in the Galactic centre has been claimed as the result of infalling unprocessed material into the central region. Such accretion has also been claimed towards the CMZ of the Milky Way through the study of isotopic enrichment (Riquelme et al. 2010). Our results show that at high resolution the degree of stellar processing of the molecular gas in the CMZ of NGC 253, as measured by carbon monoxide isotopologues, appears to be similar to that in the $\mathrm{CMZ}$ of the Galaxy.

Our ${ }^{12} \mathrm{C} /{ }^{13} \mathrm{C}$ ratio of $21 \pm 6$ is well in agreement with the value of $24 \pm 1$ in the Galactic centre, measured with the sames isotopologue transitions of CO (Langer \& Penzias 1990), while the ${ }^{16} \mathrm{O} /{ }^{18} \mathrm{O}$ ratio of $130 \pm 40$ is about half of the 250 measured there (Wilson \& Rood 1994). However, the value in NGC 253 would result in a good agreement if we extrapolate the galactocentric trend observed across the large scale disc of the Galaxy (Fig. 2 in Wilson \& Rood 1994). Similarly, the ${ }^{18} \mathrm{O} /{ }^{17} \mathrm{O}$ ratio of $4.5 \pm 0.8$ is about $50 \%$ larger than the measurements by Zhang et al. (2015) towards our Galactic centre. The smaller ${ }^{16} \mathrm{O} /{ }^{18} \mathrm{O}$ and larger ${ }^{18} \mathrm{O} /{ }^{17} \mathrm{O}$ observed ratios may point towards an actual enhancement of ${ }^{18} \mathrm{O}$ as compared to the $\mathrm{CMZ}$ of the Milky Way. This might be attributed to the fast enhancement of ${ }^{18} \mathrm{O}$ by massive stars, while ${ }^{13} \mathrm{C}$ and ${ }^{17} \mathrm{O}$ are more slowly injected by low- and intermediate-mass stars (Zhang et al. 2018). Indeed, Zhang et al. (2015) found a difference in the ${ }^{18} \mathrm{O} /{ }^{17} \mathrm{O}$ ratio of the Galactic centre to the molecular clouds in the disc, which implies differences in the gas phase injection of these isotopes.

Our result at high resolution implies that the low resolution ratios measured with single dish observations actually reflect an average global value that may include two well separated molecular components: one significantly processed by the past star formation in the nuclear region and a less processed component likely infalling from the outer disc that will presumably be feeding the future star formation in the region.

In this scenario, the recent accurate optical depth corrected ${ }^{12} \mathrm{C} /{ }^{13} \mathrm{C}$ measurement of $\sim 40$ based on $\mathrm{CN}$ observations by Henkel et al. (2014) would actually be the average of the processed material in the central region $\left({ }^{12} \mathrm{C} /{ }^{13} \mathrm{C} \sim 20\right)$ plus a molecular component with a higher ${ }^{12} \mathrm{C} /{ }^{13} \mathrm{C}$ ratio. If we consider the recovered fluxed estimated in Sect. 3.1.1, the filtered out molecular component would correspond to $\sim 70 \%$ of the ${ }^{13} \mathrm{CO}$ single dish emission, as well as $40-55 \%$ of that of ${ }^{13} \mathrm{C}^{18} \mathrm{O}$. This yields isotopic ratios for the filtered component of ${ }^{12} \mathrm{C} /{ }^{13} \mathrm{C} \sim$ $50-70$, in order to explain the global averaged single dish carbon isotopic ratio. This extended molecular component might be actually that traced by $\mathrm{CCH}$ (Martín et al. 2010), where ratios $>56$ and $>81$ were estimated based on the non-detection of ${ }^{13} \mathrm{CCH}$, and the stacked spectra of ${ }^{13} \mathrm{CCH}+\mathrm{C}^{13} \mathrm{CH}$, respectively. In fact, the observation of CN obtained as part of the ALCHEMI line survey towards NGC 253 (Martín et al., in prep.) show an optical depth well in excess of the $\tau \sim 0.2-0.5$ obtained by single dish data (Henkel et al. 2014), which would further support the fact that single dish data are indeed the result of the averaging of an optically thin extended molecular component plus a compact optically thick one. A similar calculation cannot be estimated for the extended ${ }^{16} \mathrm{O} /{ }^{18} \mathrm{O}$ ratio since the single dish global value is actually derived through assumptions on the ${ }^{12} \mathrm{C} /{ }^{13} \mathrm{C}$ ratio as explained in Sect. 4.2.

While large scale extragalactic unresolved observations might still make use of commonly assumed single dish derived ratios, high resolution observations may need to consider the multicomponent nature of the molecular clouds in the central region of galaxies. As an example, we note that the $\mathrm{HCN} / \mathrm{H}^{13} \mathrm{CN}$ and $\mathrm{HCO}^{+} / \mathrm{H}^{13} \mathrm{CO}^{+}$line ratios ranging $10-15$ obtained at $2^{\prime \prime}$ resolution (Meier et al. 2015) are actually close to the ${ }^{12} \mathrm{C} /{ }^{13} \mathrm{C}$ isotopic ration derived in this paper. Thus their derived optical depths of $\tau \sim 5-8$ for $\mathrm{HCN}$ and $\mathrm{HCO}^{+}$would actually be significantly overestimated. If we recalculate the optical depths for these two dense gas traces assuming our derived ${ }^{12} \mathrm{C} /{ }^{13} \mathrm{C} \sim 21$, it results in moderate optical depths of $\tau \sim 0.7-1.7$.

Our results also shed some doubts on the feasibility of inferring the history of stellar nucleosynthesis and the characteristics of the IMF in galaxies based on global isotopic ratios (Romano et al. 2017). Actually the molecular gas affected by the stellar nucleosynthesis in the central region might be masked or diluted by the infalling of gas from less processed regions, as it appears to be the case in NGC 253, or as result of galactic interactions. Zhang et al. (2018) recently reported a potential trend of the ${ }^{13} \mathrm{CO} / \mathrm{C}^{18} \mathrm{O}$ line ratio as a function of the infrared luminosity in a sample of extragalactic sources. This trend is claimed to be linked to the stellar IMF. In that scenario, the lowest ratios of $\sim 1$ would be the result of a top-heavy stellar IMF. However, once again this is based on a single ratio at low spatial resolution, while our study shows the significant difference between the direct measurements from the maps and the optical depth corrected values towards the selected positions (Sect. 3.2). 


\section{Conclusions}

The main results from the observations of the rarer isotopologues of carbon monoxide are the following. For the first time we present unambiguous and spatially resolved detections of the double substitution of carbon monoxide ${ }^{13} \mathrm{C}^{18} \mathrm{O}$ in the extragalactic ISM of the starburst NGC 253. Carbon and oxygen isotopologue ratios have been derived with optically thin tracers at high spatial resolution resulting in lower values than previously obtained with single dish low resolution data. Our derived ratios take into account and correct the contamination of ${ }^{13} \mathrm{C}^{18} \mathrm{O}$ by $\mathrm{C}_{4} \mathrm{H}$ and the moderate optical thickness of ${ }^{13} \mathrm{CO}$. The deduced ${ }^{12} \mathrm{C} /{ }^{13} \mathrm{C} \sim 21$ agrees with the value measured towards the centre of the Milky Way, which can be understood in terms of a similar degree of nuclear processing from stellar nucleosynthesis. Both ${ }^{16} \mathrm{O} /{ }^{18} \mathrm{O} \sim 130$ and ${ }^{18} \mathrm{O} /{ }^{17} \mathrm{O} \sim 4.5$ are well below and above those measured in the Galactic centre, respectively, which points out to ${ }^{18} \mathrm{O}$ enhancement by massive stars and a slower injection of ${ }^{13} \mathrm{C}$ by low- and intermediate-mass stars. Differences from the ratios observed with single dish telescopes appear to present evidence of a multicomponent scenario with molecular gas highly processed in the central region of NGC 253 and unprocessed gas claimed to be infalling from the outer regions of the galaxy. No obvious gradients are found as a function of the distance to the centre out to galactocentric radii of $\sim 300 \mathrm{pc}$.

Acknowledgements. SM wants to thank the valuable discussions with V. Rivilla, J. Martín-Pintado, and Laura Colzi on the results presented in this paper. KS was supported by the MOST grant 107-2119-M-001-022. This pape makes use of the following ALMA data: ADS/JAO.ALMA\#2016.1.00292.S ALMA is a partnership of ESO (representing its member states), NSF (USA) and NINS (Japan), together with NRC (Canada), MOST and ASIAA (Taiwan), and KASI (Republic of Korea), in cooperation with the Republic of Chile. The Joint ALMA Observatory is operated by ESO, AUI/NRAO and NAOJ.

\section{References}

Aladro, R., Martín, S., Riquelme, D., et al. 2015, A\&A, 579, A101 Ando, R., Nakanishi, K., Kohno, K., et al. 2017, ApJ, 849, 81 Belloche, A., Müller, H. S. P., Menten, K. M., Schilke, P., \& Comito, C. 2013, A\&A, 559, A47

Chiappini, C., Ekström, S., Meynet, G., et al. 2008, A\&A, 479, L9

Gardner, F. F., \& Whiteoak, J. B. 1979, MNRAS, 188, 331

Gardner, F. F., \& Whiteoak, J. B. 1982, MNRAS, 199, 23P

Goto, M., Usuda, T., Takato, N., et al. 2003, ApJ, 598, 1038

Henkel, C., \& Mauersberger, R. 1993, A\&A, 274, 730

Henkel, C., Wilson, T. L., \& Bieging, J. 1982, A\&A, 109, 344

Henkel, C., Mauersberger, R., Wiklind, T., et al. 1993, A\&A, 268, L17

Henkel, C., Wilson, T. L., Langer, N., Chin, Y. N., \& Mauersberger, R. 1994, in The Structure and Content of Molecular Clouds, eds. T. L. Wilson, \& K. J. Johnston, Lect. Notes Phys. (Berlin: Springer Verlag), 439, 72

Henkel, C., Asiri, H., Ao, Y., et al. 2014, A\&A, 565, A3

Ikeda, M., Hirota, T., \& Yamamoto, S. 2002, ApJ, 575, 250
Jiménez-Donaire, M. J., Cormier, D., Bigiel, F., et al. 2017, ApJ, 836, L29

Karakas, A. I., \& Lattanzio, J. C. 2014, PASA, 31, e030

Krips, M., Martín, S., Peck, A. B., et al. 2016, ApJ, 821, 112

Langer, W. D., \& Penzias, A. A. 1990, ApJ, 357, 477

Langer, W. D., Graedel, T. E., Frerking, M. A., \& Armentrout, P. B. 1984, ApJ, 277,581

Leroy, A. K., Bolatto, A. D., Ostriker, E. C., et al. 2015, ApJ, 801, 25

Limongi, M., \& Chieffi, A. 2018, ApJS, 237, 13

Loison, J. C., Wakelam, V., Gratier, P., et al. 2019, MNRAS, 485, 5777

Mangum, J. G., Darling, J., Henkel, C., et al. 2013, ApJ, 779, 33

Mangum, J. G., Ginsburg, A. G., Henkel, C., et al. 2019, ApJ, 871, 170

Martín, S., Martín-Pintado, J., Mauersberger, R., Henkel, C., \& García-Burillo, S. 2005, ApJ, 620, 210

Martín, S., Mauersberger, R., Martín-Pintado, J., Henkel, C., \& García-Burillo, S. 2006, ApJS, 164, 450

Martín, S., Aladro, R., Martín-Pintado, J., \& Mauersberger, R. 2010, A\&A, 522, A62

McMullin, J. P., Waters, B., Schiebel, D., Young, W., \& Golap, K. 2007, in Astronomical Data Analysis Software and Systems XVI, eds. R. A. Shaw, F. Hill, \& D. J. Bell, ASP Conf. Ser., 376, 127

Meier, D. S., \& Turner, J. L. 2001, ApJ, 551, 687

Meier, D. S., Walter, F., Bolatto, A. D., et al. 2015, ApJ, 801, 63

Meyer, B. S. 1994, ARA\&A, 32, 153

Milam, S. N., Savage, C., Brewster, M. A., Ziurys, L. M., \& Wyckoff, S. 2005, ApJ, 634, 1126

Mouhcine, M., Ferguson, H. C., Rich, R. M., Brown, T. M., \& Smith, T. E. 2005, ApJ, 633, 810

Müller, H. S. P., Thorwirth, S., Roth, D. A., \& Winnewisser, G. 2001, A\&A, 370, L49

Müller, H. S. P., Schlöder, F., Stutzki, J., \& Winnewisser, G. 2005, J. Mol. Struct., 742,215

Muller, S., Beelen, A., Guélin, M., et al. 2011, A\&A, 535, A103

Ott, J., Weiss, A., Henkel, C., \& Walter, F. 2005, ApJ, 629, 767

Pickett, H. M., Poynter, I. R. L., Cohen, E. A., et al. 1998, J. Quant. Spectr. Rad. Transf., 60, 883

Rekola, R., Richer, M. G., McCall, M. L., et al. 2005, MNRAS, 361, 330

Riquelme, D., Amo-Baladrón, M. A., Martín-Pintado, J., et al. 2010, A\&A, 523, A51

Röllig, M., \& Ossenkopf, V. 2013, A\&A, 550, A56

Romano, D., Matteucci, F., Zhang, Z.-Y., Papadopoulos, P. P., \& Ivison, R. J. 2017, MNRAS, 470, 401

Roueff, E., Loison, J. C., \& Hickson, K. M. 2015, A\&A, 576, A99

Sage, L. J., Henkel, C., \& Mauersberger, R. 1991, A\&A, 249, 31

Sakamoto, K., Mao, R.-Q., Matsushita, S., et al. 2011, ApJ, 735, 19

Szűcs, L., Glover, S. C. O., \& Klessen, R. S. 2014, MNRAS, 445, 4055

Turner, B. E. 1989, ApJS, 70, 539

Turner, J. L., \& Ho, P. T. P. 1985, ApJ, 299, L77

Ulvestad, J. S., \& Antonucci, R. R. J. 1997, ApJ, 488, 621

Visser, R., van Dishoeck, E. F., \& Black, J. H. 2009, A\&A, 503, 323

Wallström, S. H. J., Muller, S., \& Guélin, M. 2016, A\&A, 595, A96

Walter, F., Bolatto, A. D., Leroy, A. K., et al. 2017, ApJ, 835, 265

Wang, M., Henkel, C., Chin, Y., et al. 2004, A\&A, 422, 883

Watson, W. D. 1977, in CNO Isotopes in Astrophysics, ed. J. Audouze, Astrophys. Space Sci. Lib., 67, 105

Wilson, T. L., \& Matteucci, F. 1992, A\&ARv, 4, 1

Wilson, T. L., \& Rood, R. 1994, ARA\&A, 32, 191

Wouterloot, J. G. A., Henkel, C., Brand, J., \& Davis, G. R. 2008, A\&A, 487, 237

Zhang, J. S., Sun, L. L., Riquelme, D., et al. 2015, ApJS, 219, 28

Zhang, Z.-Y., Romano, D., Ivison, R. J., Papadopoulos, P. P., \& Matteucci, F. 2018, Nature, 558, 260 


\section{Appendix A: Error and signal to noise in line ratio maps}

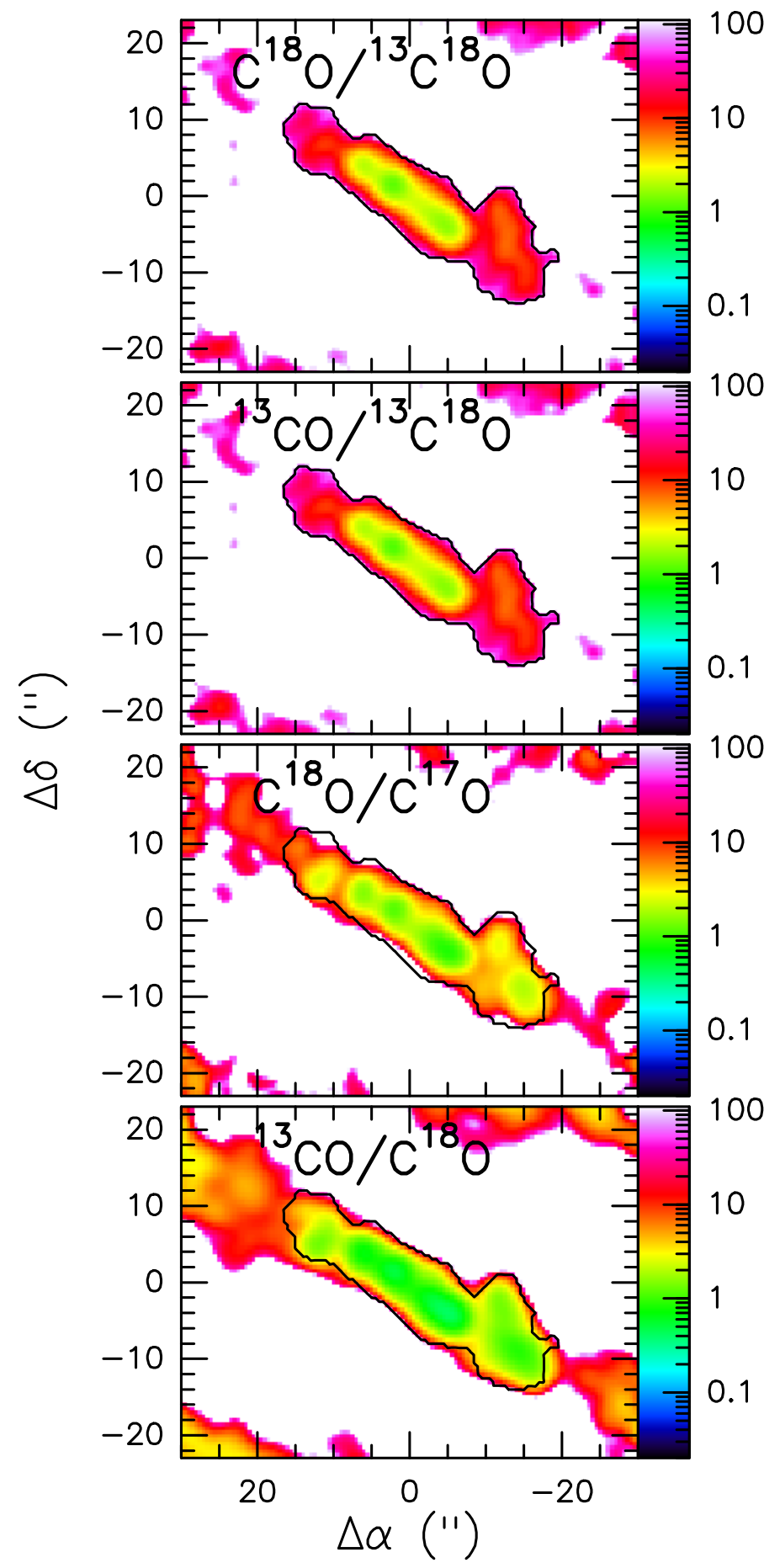

Fig. A.1. Accuracy of the ratio maps shown in Fig. 3 in percentage as defined in Eq. (A.1). The black contour is similar to that in Fig. 3.

While Fig. 1 shows the ratio $R=M_{1} / M_{2}$ between the corresponding maps $M_{1}$ and $M_{2}$, Fig. A.1 gives the ratio of the propagated error of the ratio map to the ratio map in percentage as

$\frac{\sigma_{\mathrm{R}}}{R}(\%)=\frac{100}{R} \sqrt{\left(\frac{\sigma_{M_{1}}}{M_{2}}\right)^{2}+\left(\frac{\sigma_{M_{2}} M_{1}}{M_{2}^{2}}\right)^{2}}$
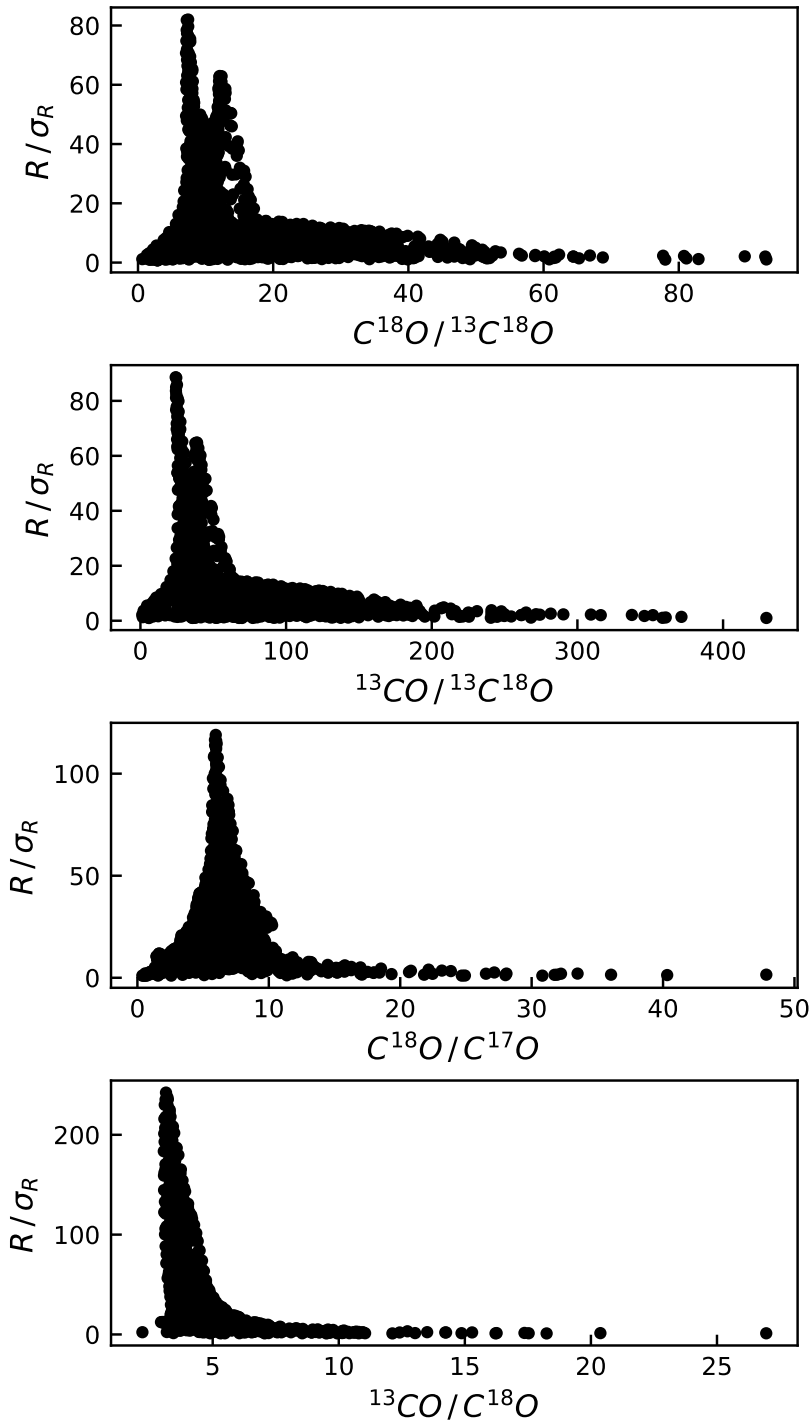

Fig. A.2. Signal to noise ratios per pixel in the ratio maps of Fig. 3 as a function of the line ratio.

where we observe how the central region, as expected, has errors of $\sim 1 \%$ rising to a few $10 \%$ towards the edge of the region considered (see Sect. 3.1.2).

Figure A.2, on the other hand, shows the signal to noise ratio per pixel of the ratio maps $\left(R / \sigma_{\mathrm{R}}\right)$ as a function of the ratio. The effect of line contamination by $\mathrm{C}_{4} \mathrm{H}$ (Sect. 3.2.1) in the $\mathrm{C}^{18} \mathrm{O} /{ }^{13} \mathrm{C}^{18} \mathrm{O}$ and ${ }^{13} \mathrm{CO} /{ }^{13} \mathrm{C}^{18} \mathrm{O}$ is clearly seen as the double peak structure and a broad tail both at high signal to noise as a result of the different distributions between the $\mathrm{CO}$ isotopologues and $\mathrm{C}_{4} \mathrm{H}$. This is different from what is observed in the $\mathrm{C}^{18} \mathrm{O} / \mathrm{C}^{17} \mathrm{O}$ and ${ }^{13} \mathrm{CO} / \mathrm{C}^{18} \mathrm{O}$ ratios which show a single peaked distribution. The effect of the significant ${ }^{13} \mathrm{CO}$ optical depth is evidenced by the ${ }^{13} \mathrm{CO} / \mathrm{C}^{18} \mathrm{O}$ distribution being skewed towards lower values, which is different from what we observe in the optically thin ratio $\mathrm{C}^{18} \mathrm{O} / \mathrm{C}^{17} \mathrm{O}$ more symmetrical distribution. Similarly the two high signal to noise components in ${ }^{13} \mathrm{CO} /{ }^{13} \mathrm{C}^{18} \mathrm{O}$ are also pushed together by the effect of optical depth. See Sect. 4.1 for further discussion of both optical depth and line contamination effects on ratio maps based on the differences observed with the ratios from spectral analysis. 


\section{Appendix B: Selected positions: Comparison to other works}

Positions in this work are based on observed maxima in the $\mathrm{C}^{18} \mathrm{O}$ channel maps (see Sect. 3.2) and not from those in previous high resolution studies (Sakamoto et al. 2011; Meier et al. 2015; Ando et al. 2017; Leroy et al. 2015; Walter et al. 2017). Figure B.1 shows the positions selected in this paper compared to those by Meier et al. (2015) aiming at sampling different regions in NGC $253 \mathrm{CMZ}$, those of Sakamoto et al. (2011) identifying the brightest spots in the integrated intensity maps, and those of the high resolution study by Ando et al. (2017) identifying the peak continuum sources in the very inner region. The different angular resolutions of these studies in the literature are also shown in this Fig. B.1.

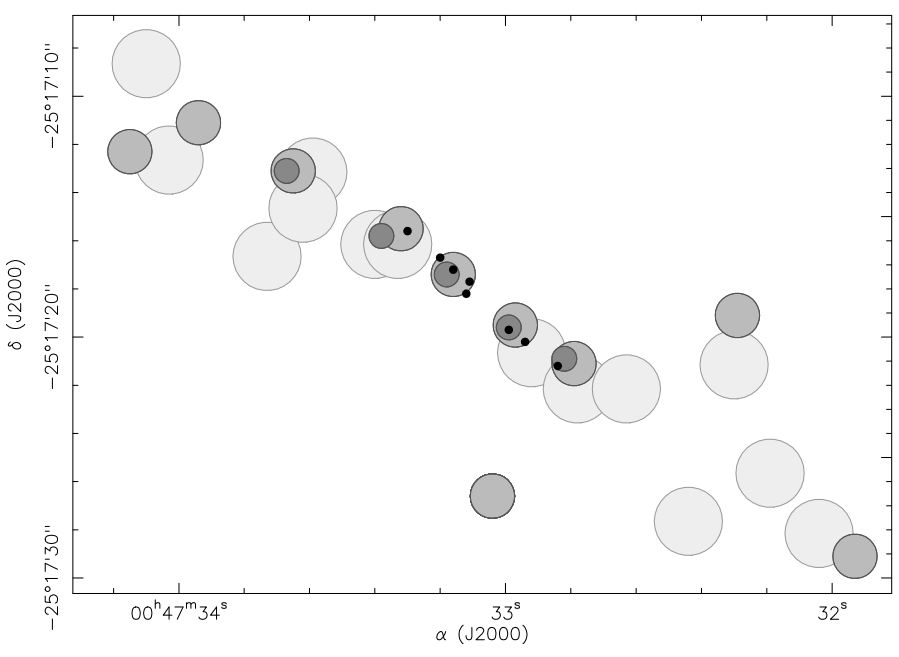

Fig. B.1. Location of the positions analysed in this paper (3", light grey circles), compared to the positions from Meier et al. (2015; 2", grey circles), Sakamoto et al. (2011; 1.1", dark grey circles), and Ando et al. (2017; $0.37^{\prime \prime}$, black dots). The size of the circles represent the resolution of the observations in these studies. 